\title{
A Norm Principle for class groups of reductive group schemes over Dedekind rings
}

\author{
Nguyêñ Quôć Thăńg *
}

\begin{abstract}
We discuss and prove some results on Corestriction principle for non-abelian étale cohomology and Norm principle for class groups of reductive group schemes over Dedekind rings in global fields.

AMS Mathematics Subject Classification (2000): Primary 11E72, 14F20, 14L15; Secondary 14G20, 14G25, 18G50, 20G10. Key words: Non-abelian cohomology. Rerductive group schemes. Norm Principle. Corestriction map.
\end{abstract}

\section{Introduction.}

The well-known notion of class group of a global field plays an important role in number theory in general and in the arithmetic of global fields in particular. Its natural generalization to algebraic groups also turns out to be an important notion in the study of arithmetic of algebraic groups over local and global fields.

Since algebraic groups under consideration may be not commutative, the best we can afford is to associate to a given linear algebraic group $G_{k}$ defined over a global field $k$ a set of double cosets, called the class set of $G_{k}$. However, this set is not an invariant in the $k$-isomorphism class of $G$. To remedy the situation, one may consider a model of $G$ over a Dedekind ring in $k$. We consider more generally the class set of a given flat affine group scheme $G$ of finite type defined over Dedekind ring $A$ with smooth generic fiber $G_{k}$ over the global quotient field $k$ of $A$. Let $X=\operatorname{Spec}(A), \eta \in X$ the generic point of $X, S$ a finite subset of $X_{0}:=X \backslash\{\eta\}$. The ring $A(S)$ of $S$-adèles is defined as

$$
\mathbf{A}(S):=\prod_{v \in X_{0} \backslash S} A_{v} \times \prod_{v \in S} k_{v}
$$

where $k_{v}$ (resp. $A_{v}$ ) is the completion of $k$ (resp. $A$ ) in the $v$-adic topology. We denote by $\mathbf{A}=$ ind.lim $_{S} \mathbf{A}(S)$ the adèle ring of $k$ (with respect to $A$ !). Recall that (see e. g. [B], [PlR], Chap. VIII, in the case of linear algebraic groups and [Gi1, [Gi2], [Ha], [Ni1] in the

\footnotetext{
*Institute of Mathematics, Vietnam Academy of Sciences and Technology, 18 Hoang Quoc Viet, Hanoi Vietnam. Supported in part by NAFOSTED, VIASM, Abdus Salam I. C. T. P. (through (S.I.D.A.)) and Max Planck Institut für Mathematik, Bonn. E-mail : nqthang@math.ac.vn
} 
case of group schemes) the $S$-class set, of $G$ with respect to a finite set $S$ of primes of $A$ (denoted by $C l_{A}(S, G)$ ), and the class set of $G$ (denoted by $C l_{A}(G)$ ), is the set of double classes

$$
C l_{A}(S, G)=G(\mathbf{A}(S)) \backslash G(\mathbf{A}) / G(k),
$$

and

$$
C l_{A}(G)=G(\mathbf{A}(\emptyset)) \backslash G(\mathbf{A}) / G(k),
$$

respectively. (Here $G(k)$ is embedded diagonally into $G(\mathbf{A})$. Another, more familiar notation for $C l_{A}(G)$ using the set of infinite primes is given in the last section.) The important fact is that these sets are invariant in the class of $A$-isomorphism of $G$. It may happen that $C l_{A}(S, G)$ (resp. $C l_{A}(G)$ has a natural group structure (i.e. inherited from that of $G(\mathbf{A})$ ). In this case it is denoted by $\mathcal{G} C l_{A}(S, G)$ (resp. $\mathcal{G} C l_{A}(G)$ ). (By convention, in the case of global function field $k$, we assume that $k$ is the field of rational functions of a smooth irreducible affine curve $C$ defined over some finite field $\mathbf{F}_{q}$, and by convention, the ring of integers of $k$ is the ring of $\mathbf{F}_{q}$-regular functions of $C$.)

Theorem. (Norm principle for $S$-class groups of algebraic groups.)

Let $k$ be a global field, A the ring of integers of $k, G$ a reductive A-group scheme of finite type and $L / k$ a finite separable extension. Assume that for a finite set $S$ of primes of $k$, containing the set $\infty$ of archimedean primes, and for the derived subgroup $G^{\prime}=[G, G]$ of $G$, the topological group $\prod_{v \in S} G^{\prime}\left(k_{v}\right)$ is non-compact. Let $S^{\prime}$ be the (finite) set of all non-equivalent valuations of $L$ which are extensions of those in $S$ to $L$. Then for $A^{\prime}$ the integral closure of $A$ in $L$, the class set $C l_{A^{\prime}}\left(S^{\prime}, G\right)$ has a natural structure of finite abelian group, and we have a norm homomorphism, functorial in $G, A$

$$
N_{A^{\prime} / A}: \mathcal{G} C l_{A^{\prime}}\left(S^{\prime}, G\right) \rightarrow \mathcal{G C l}_{A}(S, G),
$$

such that for $A^{\prime}=A, N_{A^{\prime} / A}=i d$, and for a tower of finite separable extensions $K / L / k$, with obvious notations $S^{\prime \prime} / S^{\prime} / S$, we have

$$
N_{A^{\prime \prime} / A}=N_{A^{\prime} / A} \circ N_{A^{\prime \prime} / A^{\prime}}
$$

In fact, we give two proofs of this theorem. A short presentation of the results obtained here was announced before (see [T7] (appeared in 2006), and also in preprint form [T8] (appeared in 2007)). Quite recently, after [T7], [T8] had been done, there appeared interesting papers and thesis by C. Demarche ([Dem1] (2011), [Dem2] (2009)), where among other things, he gave another proof for our Theorem above. His proof is based on some results of his theory of approximation for complexes of tori, but only in the case of number fields, whereas our result holds true over any global field. So our paper can be considered as a complement to the work by Demarche. Later on, there was some extension to a more general base by Gonzales-Aviles [GA] (2013), with different technique of the proofs. One of our main tools is Theorem 3.2, which we hope can be further strengthend to prove the existence of the norm map in a more general case, to which we hope to return later on. 


\section{Some preliminary results}

We refer the reader to [SGA 3] for standard notation and terminology used below.

1.1. Induced tori. We need the following analogs of some results proved in [Bo], [Ko], [T2], [T3]. First, we recall the important notion of induced (or quasi-trivial) tori (see [Ha], pp. 171 - 172, especially [CTS2], Section 1).

For a noetherian domain $R$ with quotient field $k$, such that $\operatorname{Spec}(R)$ is geometrically unibranch and connected, we recall that (cf. [SGA 3], Exp. X, Remark 5.15, Théorème 5.16) for an $R$-torus $T$ there is a finite étale extension $S / R$, with quotient field $k^{\prime}$ such that $T_{S}$ is $S$-isomorphic to $\mathbf{G}_{m}^{r}$ for some $r$. We may assume that $k^{\prime} / k$ is a finite Galois extension, and that $S / R$ is also a Galois extension with the same Galois group $\Gamma:=\operatorname{Gal}(S / R)=$ $\operatorname{Gal}\left(k^{\prime} / k\right)$. Denote by $X_{S}(T):=\operatorname{Hom}_{S}\left(T_{S}, \mathbf{G}_{m}\right)$ the character group, which is a $\Gamma$-module and it determines the $R$-group scheme $T$ up to a unique $R$-isomorphism ([SGA 3], Exp. X, Théorème 1.1). $T$ is called $R$-induced (or $R$-quasi-trivial) if there are a subgroup $\Gamma_{0} \subset \Gamma$ and a $\Gamma$-submodule $X_{0} \subset X_{S}(T)$ such that $\Gamma_{0}$ acts trivially on $X_{0}$ and

$$
X_{S}(T)=\bigoplus_{\sigma \in \Gamma / \Gamma_{0}} \sigma\left(X_{0}\right)
$$

Equivalently, an induced $R$-torus $T$ is $R$-isomorphic to a finite direct product of $X$-tori of the form $R_{S_{i} / R}\left(\mathbf{G}_{m}\right)$ (cf. also [CTS2], Section 1).

1.2. z-extensions. As in the case of fields, for a ring $R$ as above, and an exact sequence $1 \rightarrow Z \rightarrow H \rightarrow G \rightarrow 1$ of reductive $R$-group schemes, with $Z$ an $R$-torus (cf. [SGA 3], Exp. XXII, Sec. 4.3.3, for the corresponding notions), we say (after Langlands) that $H$ is a z-extension of $G$ if $Z$ is an induced $R$-torus and the derived subgroup of $H$ is simply connected. Now, if $x \in \mathrm{H}^{1}(S, G)$, we say that a $z$-extension $H \rightarrow G$ (over $R$ ) is $x$-lifting if $x \in \operatorname{Im}\left(\mathrm{H}^{1}\left(S, H_{S}\right) \rightarrow \mathrm{H}^{1}\left(S, G_{S}\right)\right)$.

Note that the crossed-diagram construction by Ono (used in [Ha1]) also relates to the notion of $z$-extensions used by Langlands. We fix a noetherian domain $R$ as in 1.1 and consider in this section the category $\mathcal{G} S c h_{R}$ of flat affine group schemes over $\operatorname{Spec}(R)$ of finite type. The existence of $z$-extensions in the case of fields was proved in Borovoi [Bo] and Kottwitz [Ko] (in the case of fields of characteristic 0) and extended to more general case in [T6], Lemma 2.2.1. In fact, some conditions were omitted in loc.cit, and the referee pointed out several points in the proof of (loc.cit) which need to be clarified and we take a chance to present some corrections and modifications here. (In fact, only the existence of $z$-extension is what we need later on in Section 4.)

1.2.1. Lemma. For $R$ as in 1.1, and $G$ a connected reductive $R$-group, there exists a $z$-extension $1 \rightarrow Z \rightarrow H \rightarrow G \rightarrow 1$.

We give below a correct formulation of Lemma 2.2.1 of [T6], from which Lemma 1.2.1 follows. We first need the following assertions. 
1.2.2. Lemma. (Cf. [SGA3, Exp. X, 1.3, 5.15, 5.16]) Let $S$ be a locally noetherian, connected and geometrically unibranch scheme. Then any S-group scheme $H$ of multiplicative type and of finite type over $S$ is isotrivial, i.e., $H$ becomes split (diagonalizable) over a finite surjective étale cover $S$ ' of $S$.

It is known that if $H$ is an isotrivial group scheme of multiplicative type over a connected scheme $S$, then $H$ is split over a finite étale connected cover $S^{\prime} \rightarrow S$, which is a finite Galois cover in the sense of [SGA1, Exp. V, 2.8].

Let $G$ be a reductive $R$-group. Denote by $\operatorname{rad}(G)$ the radical of $G, \tilde{G}$ the simply connected covering of the derived subgroup $G^{\prime}:=[G, G]$ of $G$,

$$
\pi: \tilde{G} \times_{\operatorname{Spec}(R)} \operatorname{rad}(G) \rightarrow G^{\prime} \times_{\mathrm{Spec}(R)} \operatorname{rad}(G) \rightarrow G
$$

the composition of central isogenies (cf. [SGA 3], Exp. XXII, Prop. 6.2.4). Let $A=\operatorname{Ker}(\pi)$. The following lemma is the corrected version of [T6, Lem. 2.2.1] and is due to Borovoi and/or Kottwitz (see [Bor], Sec. 3, [Ko1], [Ko2]) in the case $S, R$ are fields. The method of proof is similar, but for the self-containedness and convenience of readers, we give them here.

1.2.3. Lemma. Let $R$ be a ring such that $\operatorname{Spec}(R)$ is a locally noetherian, connected and geometrically unibranch.

a) Let $F$ be a finite flat $R$-group scheme. Then there exist a Galois extension $S / R$ which splits $F$ and an induced $R$-torus $Z$ which is $R$-isomorphic to $\operatorname{Res}_{S / R}\left(\mathbf{G}_{m}\right)^{n}$ for some $n$ with an embedding of $R$-group schemes $F \hookrightarrow Z$.

b) Let $G$ be a $R$-reductive group, $\pi, A$ be as above, where $A$ is split over a finite étale connected extension $S / R$. Then there exists a z-extension $1 \rightarrow Z \rightarrow H \rightarrow G \rightarrow 1$ over $R$, such that $Z \simeq \operatorname{Res}_{S^{\prime} / R}\left(\mathbf{G}_{m}\right)^{n}$ for some $n$ and Galois extension $S^{\prime} / R$ which contains $S$.

c) Let $G$ be a reductive $R$-group, $S^{\prime} / S / R$ finite étale connected covers of $R, x \in \mathrm{H}_{e t}^{1}\left(S^{\prime} / S, G\right):=$ $\operatorname{Ker}\left(\mathrm{H}_{e t}^{1}(S, G) \rightarrow \mathrm{H}_{e t}^{1}\left(S^{\prime}, G\right)\right)$. Then the exists a z-extension $1 \rightarrow Z \rightarrow H \rightarrow G \rightarrow 1$ over $R$, which is $x$-lifting.

Proof. a) Under the new assumption on the $\operatorname{ring} R$ and by using 1.2.1, the arguments used in the proof of a) and b) given in [T6, p.94-95] holds true. Since the argument is short, we repeat it here.

By the choice of $R$, by [SGA 3, Exp. X, Corol. 1.2], there is an anti-equivalence between the category of $R$-multiplicative groups and the category of continuous $\Pi$-modules (i.e., the stabilizer in $\Pi$ of any point of the module is open), where $\Pi=\pi_{1}(\operatorname{Spec}(R), \psi)$ the fundamental group of $\operatorname{Spec}(R)$ in the sense of Grothendieck (cf. [SGA 1], Exp. V) with respect to a geometric point $\psi: \operatorname{Spec}\left(k_{s}\right) \rightarrow \operatorname{Spec}(R)$. Here $k_{s}$ denotes a separable closure of the quotient field $k$ of $R$. In particular, $\Gamma$ is a finite quotient group of $\Pi$. The corresponding functor is given by character group on the fiber at geometric point

$$
H \mapsto M_{H}:=\operatorname{Hom}_{g r}\left(H_{\psi}, \mathbf{G}_{m, \psi}\right) .
$$

In our case, if $F$ corrresponds to a $\Pi$-module $M_{F}$, then $M_{F}$ is a finite $\mathbf{Z}[\Gamma]$-module, thus 
there is a surjective homomorphism of $\Gamma$-modules $M_{B} \rightarrow M_{F}$, where $M_{B}$ is a free $\mathbf{Z}[\Gamma]$ module $\mathbf{Z}[\Gamma]^{n}$, where $n=\operatorname{Card}\left(M_{F}\right)$, considered as a $\mathbf{Z}[\Pi]$-module, with trivial action of $\operatorname{Ker}(\Pi \rightarrow \Gamma)$ on $M_{B}$. The $R$-torus $B$ corresponding to $M_{B}$ has the form $\operatorname{Res}_{S^{\prime} / R}\left(\mathbf{G}_{m}\right)^{n}$. Due to the surjectivity of the homomorphism $M_{B} \rightarrow M_{F}$, the corresponding $R$-morphism $F \rightarrow B$ is injective.

b) By a), there exists an induced $R$-torus $Z$ such that $A \hookrightarrow Z$. We set

$$
H=\left(\tilde{G} \times_{\operatorname{Spec}(R)} \operatorname{rad}(G) \times_{\operatorname{Spec}(R)} Z\right) / A,
$$

where $A$ is embedded into the product in an obvious way. Then $G=(\tilde{G} \times \operatorname{Spec}(R) \operatorname{rad}(G)) / A$, and the obvious map $H \rightarrow G$ is clearly surjective. Its kernel is $Z$, and we have a $z$-extension as required.

c) We use the $z$-extension obtained in b). We may assume that $S^{\prime} / R$ is a Galois extension with Galois group $\Gamma$. Then we have the following commutative diagram

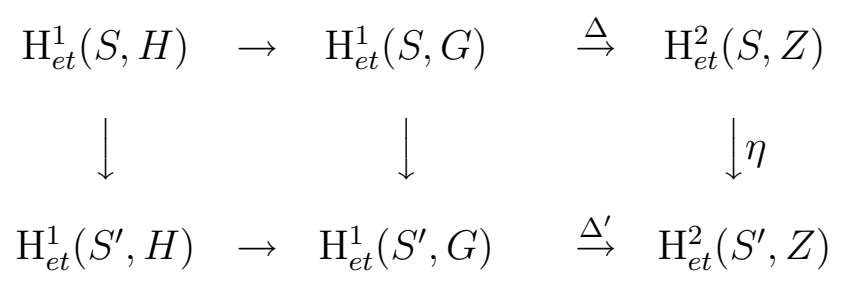

where all lines are exact, the vertical arrows are restriction maps, and the maps $\Delta, \Delta^{\prime}$ are coboundary maps (see [Gir], Chap. IV, Sec. 3.5). Setting $Z=\operatorname{Res}_{S^{\prime} / S}(T)$, where $T:=\left(\mathbf{G}_{m}\right)_{S^{\prime}}^{n}$. Then $Z=\operatorname{Res}_{S^{\prime} / S}\left(Z_{1}\right)$, where $Z_{1}(U):=T\left(S \otimes_{R} U\right)$ for any $S^{\prime}$-algebra $U$. Then one checks that $\mathrm{H}_{e t}^{2}(S, Z) \simeq \mathrm{H}_{e t}^{2}\left(S^{\prime}, Z_{1}\right)$ (see 1.3.4.1) and due to the diagonal embedding

$$
Z_{1, S^{\prime}} \hookrightarrow \operatorname{Res}_{S^{\prime} / S}\left(Z_{1}\right)_{S^{\prime}} \simeq \prod_{\gamma \in \Gamma} Z_{1, S^{\prime}}
$$

it implies that the induced map

$$
\gamma^{\prime}: \mathrm{H}_{e t}^{2}(S, Z)=\mathrm{H}_{e t}^{2}\left(S^{\prime}, Z_{1}\right) \rightarrow \mathrm{H}_{e t}^{2}\left(S^{\prime}, \operatorname{Res}_{S^{\prime} / S}\left(Z_{1}\right)\right)
$$

is just embedding, thus also injective. Further the rest of the proof remains the same as in [T6].

(In [T6], the arrow $\eta$ on p. 95, line 12 from the bottom should be shifted to the right to make a map $\eta: \mathrm{H}_{e t}^{2}(S, Z) \rightarrow \mathrm{H}_{e t}^{2}(S, Z)$.)

Since the rings of integers of global fields satisfy the condition of Lemma 1.2.3, Lemma 1.2.1 follows from 1.2.3.

1.2.4. Other corrections to [T6]. We take a chance to make some corrections to [T6]. Therefore the following assumptions should be added in order the results be valid : 
1) All the rings under consideration in [T6] are assumed to be connected, noetherian and geometrically unibranch. (This is needed if we use Grothendieck theory in [SGA3, Exp. IX, $\mathrm{X}]$ to make sure the existence of $z$-extensions.)

2) P. 112, line (-10): The numbering 4.7 (resp. 4.8, 4.9) should be changed to 4.8 (resp. 4.9, 4.10).

\subsection{Deligne hypercohomology and abelianized cohomology.}

1.3.1. Deligne hypercohomology. (See [De], [Br], Section 4.) In [De], Sec. 2.4, Deligne has associated to each pair $f: G_{1} \rightarrow G_{2}$ of algebraic groups defined over a field $k$, where $f$ is a $k$-morphism, a category $\left[G_{1} \rightarrow G_{2}\right]$ of $G_{2}$-trivialized $G_{1}$-torsors, and certain hypercohomology sets denoted by $\mathbf{H}^{i}\left(G_{1} \rightarrow G_{2}\right)$, which fits into an exact sequence involving $G_{1}(k), G_{2}(k)$ and their first Galois cohomologies. In many important cases, the above category appears to be a strictly commutative Picard category (loc.cit). In [De], p. 276, there was also an indication that the construction given there can be done for sheafs of groups over any topos. Thus in [De], Section 2.4, there was defined the hypercohomology sets $\mathbf{H}_{r}^{i}\left(G_{1} \rightarrow G_{2}\right)$ for $i=-1,0$, where $r$ stands for étale or flat topology. (To be consistent, we use the notations of [Bo] and [Br], Section 4, while in [De], the degree of the hypercohomology sets corresponding to $G_{1} \rightarrow G_{2}$ is shifted.) In particular, the existence of a norm map (i.e., the validity of Corestriction principle) for hypercohomology in degree 0 in the case of local and global fields was first proved by Deligne [De], Prop. 2.4.8.

Later on, Borovoi in [Bo] (resp. Breen in [Br], Section 4, gave a detailed exposition and extension of such hypercohomology theory over fields of characteristric 0 (resp. for arbitrary site). Namely, in [Bo] (resp. [Br]), there was defined also the set $\mathbf{H}^{1}\left(G_{1} \rightarrow G_{2}\right.$ ) (resp. $\mathbf{H}^{1}\left(\mathcal{T}, G_{1} \rightarrow G_{2}\right)$, where the setting in $[\mathrm{Br}]$ works over any topos $\left.\mathcal{T}\right)$. In the particular case, when the base scheme is the spectrum of a field of characteristic 0, the Breen theory coincides with the one given by Borovoi $[\mathrm{Bo}])$.

1.3.2. Breen cohomology theory. (Cf. [Br], Sections 3, 4.) Recall the following general results due to Breen [Br], Section 4 related to $\mathbf{H}^{i}$ of a crossed module. Let $\partial: G_{1} \rightarrow G_{0}$ be a crossed module in a topos $\mathcal{T}$. Then there exists a uniquely determined simplicial group $\mathcal{G}$ in $\mathcal{T}$ associated to $\partial: G_{1} \rightarrow G_{0}$. Together with $\mathcal{G}$, one defines also the abelian (simplicial) loop group $\Omega \mathcal{G}$ in $\mathcal{T}$, and the (simplicial) classifying group $B \mathcal{G}$, which are defined by $(B \mathcal{G})_{i}:=B\left(\mathcal{G}_{i}\right)$. To define cohomology of crossed modules, one defines first the loop space $\Omega \mathcal{G}$ and the classifying space $B \mathcal{G}$ of $\mathcal{G}$, the derived category $D_{\bullet}(\mathcal{T})$ of the category of simplical objects of $\mathcal{T}$, obtained by localizing the (homotopies) quasi-isomorphisms. Then let $e$ be the final object of $D_{\bullet}(\mathcal{T})$ and one defines the cohomology of $T$ with values in the crossed module $\partial: G_{1} \rightarrow G_{0}$ in degrees $-1,0,1$ (see loc. cit. for details) by

$$
\begin{aligned}
& \mathrm{H}^{-1}\left(\mathcal{T}, G_{1} \rightarrow G_{0}\right):=\operatorname{Hom}_{D_{\bullet}(\mathcal{T})}(e, \Omega \mathcal{G}), \\
& \mathrm{H}^{0}\left(\mathcal{T}, G_{1} \rightarrow G_{0}\right):=\operatorname{Hom}_{D_{\bullet}(\mathcal{T})}(e, \mathcal{G}),
\end{aligned}
$$




$$
\mathrm{H}^{1}\left(\mathcal{T}, G_{1} \rightarrow G_{0}\right):=\operatorname{Hom}_{D \bullet(\mathcal{T})}(e, B \mathcal{G}) .
$$

Then we have the following exact sequence (see $[\mathrm{Br}]$, Section 4)

$$
\begin{aligned}
1 & \rightarrow \mathrm{H}^{-1}\left(\mathcal{T}, G_{1} \rightarrow G_{0}\right) \rightarrow \mathrm{H}^{0}\left(\mathcal{T}, G_{1}\right) \rightarrow \mathrm{H}^{0}\left(\mathcal{T}, G_{0}\right) \\
& \rightarrow \mathrm{H}^{0}\left(\mathcal{T}, G_{1} \rightarrow G_{0}\right) \rightarrow \mathrm{H}^{1}\left(\mathcal{T}, G_{1}\right) \rightarrow \mathrm{H}^{1}\left(\mathcal{T}, G_{0}\right) \\
& \rightarrow \mathrm{H}^{1}\left(\mathcal{T}, G_{1} \rightarrow G_{0}\right) .
\end{aligned}
$$

1.3.3. Now, for a noetherian domain $\operatorname{ring} A$, in the particular case of $\operatorname{Spec}(A)$, by $[\mathrm{Br}]$, 4.2.2, we may also define the abelianization maps

$$
a b_{G, r}^{i}: \mathrm{H}_{r}^{i}(A, G) \rightarrow \mathbf{H}_{r}^{i}(\tilde{G} \rightarrow G):=\mathbf{H}_{r}^{i}(A, \tilde{G} \rightarrow G),
$$

for a reductive $A$-group scheme $G$, where $r$ stands for "ét" or "flat" (="fppf"), $\tilde{G}$ is the simply connected semisimple $A$-group scheme, which is the universal covering of $G^{\prime}:=[G, G]$, the semisimple part of $G$, and $i=0,1$. and $\mathcal{T}_{r}$ is the corresponding small étale site (resp. big fppf site). In fact, it has been proved in [De], Section 2.4 (and 2.7), that if $\tilde{Z}$ (resp. $Z$ ) is the center of $\tilde{G}$ (resp. of $G$ ), and $\tilde{T}$ (resp. $T$ ) is a maximal $A$-torus of $\tilde{G}$ (resp. $G$ ), with $f^{-1}(T)=\tilde{T}$, then there are an equivalence of categories $[\tilde{Z} \rightarrow Z] \simeq[\tilde{G} \rightarrow G]$, and quasi-isomorphisms of complexes

$$
(\tilde{Z} \rightarrow Z) \simeq(\tilde{T} \rightarrow T) \simeq(\tilde{G} \rightarrow G) .
$$

One defines $\mathrm{H}_{a b, r}^{i}(A, G):=\mathbf{H}_{r}^{i}(\tilde{G} \rightarrow G)\left(=\mathbf{H}_{r}^{i}(\tilde{Z} \rightarrow Z)\right)$ and call it the abelianized cohomology of degree $i$ of $G$ (in the corresponding topos; here $r$ stands for "ét" or "fppf" = "flat", (wherever they make sense) For $i=0$, it is a group homomorphism. Since $\tilde{Z}$ and $Z$ are commutative, so the resulting cohomology sets $\mathbf{H}_{r}^{i}(A, \tilde{Z} \rightarrow Z)$ (wherever they make sense), have natural structure of abelian groups. In the particular case, we have the following exact sequence, which is functorial in $A$

$$
\begin{aligned}
1 \rightarrow \mathbf{H}_{a b, e t}^{-1}(A, G) & \rightarrow \mathrm{H}_{e t}^{0}(A, \tilde{G}) \rightarrow \mathrm{H}_{e t}^{0}(A, G) \stackrel{a b_{G, e t}^{0}}{\rightarrow} \mathbf{H}_{e t}^{0}(A, \tilde{G} \rightarrow G) \rightarrow \\
& \rightarrow \mathrm{H}_{e t}^{1}(A, \tilde{G}) \rightarrow \mathrm{H}_{e t}^{1}(A, G) \stackrel{a b_{G, e t}^{1}}{\rightarrow} \mathbf{H}_{e t}^{1}(A, \tilde{G} \rightarrow G) .
\end{aligned}
$$

1.3.4. Corestriction maps. Let $A$ be a commutative domain, and let $G$ be a reductive $A$-group scheme. Denote by $G^{\prime}$ the derived subgroup scheme of $G, \tilde{G}$ the simply connected covering of $G^{\prime}, \bar{G}$ the adjoint group scheme of $G$ (see [SGA 3], Exp. XXII, 4.3.3), $\tilde{F}:=\operatorname{Ker}(\tilde{G} \rightarrow \bar{G}), F:=\operatorname{Ker}\left(\tilde{G} \rightarrow G^{\prime}\right)$ and let $\tilde{Z}=\operatorname{Cent}(\tilde{G}), Z=\operatorname{Cent}(G)$ be the corresponding centers. First we have the following (cf. also [Gi1, Sec. 0] or [T6, Prop. 2.1]).

1.3.4.1. Proposition. (Cf. [CTS, Sec. 0.4]) a) Let $p: Y \rightarrow X$ be a finite étale cover of connected scheme $X$, and let $G$ be an affine group scheme over $Y$. Then we have canonical isomorphisms

$$
\varphi_{i}: \mathrm{H}_{e t}^{i}\left(X, R_{Y / X}(G)\right) \simeq \mathrm{H}_{e t}^{i}(Y, G)
$$


for all $i \geq 0$, where $i=0,1$ if $G$ is a non-abelian group.

b) If $Y$ is as above, and if $G$ is commutative affine group over $X$, then there exists a functorial corestriction homomorphism

$$
\operatorname{Cores}_{Y / X}: \mathrm{H}_{e t}^{i}\left(Y, p^{*} G\right) \rightarrow \mathrm{H}_{e t}^{i}(X, G) \text {, for all } i \geq 0,
$$

such that if $Y=X, f=i d$, then $\operatorname{Cores}_{Y / X}=i d$, and if $f^{\prime}: Y^{\prime} \rightarrow Y$ is finite and étale, then Cores $_{Y / X} \circ$ Cores $_{Y^{\prime} / Y}=$ Cores $_{Y^{\prime} / X}$.

1.3.5. Corestriction principle. For any smooth commutative $A$-group scheme $T$ and each finite étale extension $A^{\prime} / A$, as we have seen, there is a functorial homomorphism

$$
\text { Cores }_{A^{\prime} / A, T}: \mathrm{H}_{e t}^{i}\left(A^{\prime}, T_{A^{\prime}}\right) \rightarrow \mathrm{H}_{e t}^{i}(A, T),
$$

where $T_{A^{\prime}}=T \times_{A} A^{\prime}$. Assume that we have a morphism of cohomology functors $f$ : $\left(A \mapsto \mathrm{H}_{e t}^{i}(A, G)\right) \rightarrow\left(A \mapsto \mathrm{H}_{e t}^{j}(A, T)\right)$ (resp. $g:\left(A \mapsto \mathrm{H}_{e t}^{j}(A, T)\right) \rightarrow\left(A \mapsto \mathrm{H}_{e t}^{i}(A, G)\right)$, where $G$ is non-commutative, thus a system of maps $\left.f_{A}: \mathrm{H}_{e t}^{i}(A, G)\right) \rightarrow \mathrm{H}_{e t}^{j}(A, T)$ (resp. $\left.\left.g_{A}: \mathrm{H}_{e t}^{j}(A, T)\right) \rightarrow \mathrm{H}_{e t}^{i}(A, G)\right)$.

We say that Corestriction Principle holds for the image of $f$ (resp. kernel of $g$ ) with respect to the extension $A^{\prime} / A$, if $\operatorname{Cores}_{A^{\prime} / A, T}\left(\operatorname{Im}\left(f_{A^{\prime}}\right)\right) \subset \operatorname{Im}\left(f_{A}\right)\left(\operatorname{resp} . \operatorname{Cores}_{A^{\prime} / A, T}\left(\operatorname{Ker}\left(g_{A^{\prime}}\right)\right) \subset\right.$ $\left.\operatorname{Ker}\left(g_{A}\right)\right)$.

If it holds for any finite étale extension $A^{\prime} / A$, we say Corestriction principle holds for the image of $f$ (resp. kernel of $g$ ).

Finally, if $\operatorname{Cores}_{A^{\prime} / A, T}\left(\left\langle\operatorname{Im}\left(f_{A^{\prime}}\right)\right\rangle\right) \subset\left\langle\operatorname{Im}\left(f_{A}\right)\right\rangle\left(\operatorname{resp} . \operatorname{Cores}_{A^{\prime} / A, T}\left(\left\langle\operatorname{Ker}\left(g_{A^{\prime}}\right)\right\rangle\right) \subset\left\langle\operatorname{Ker}\left(g_{A}\right)\right\rangle\right)$. where $\langle Q\rangle$ denotes the subgroup generated by $Q$, we say the Weak Corestriction principle holds for the image of $f$ (resp. kernel of $g$ ).

In [T7], [T8], we prove the validity of such principle under some restriction on domains $A$ and its field of fractions $k$.

1.3.6. In the case $A$ is a local or global field of characteristic 0 , it is known that there exists functorial corestriction homomorphisms for $\mathrm{H}_{a b, e t}^{i}(A, G), i \geq 0$ (which follows from [De], Sec. 2.4.3, cf. also [Pe], Sec. 3, [T1], Theorem 2.5). It can also be extended to the case of positive characteristic ([T3], Section 3, Theorem B), where instead of Galois cohomology, we use flat cohomology. However, in general (étale or flat) case, it is not clear whether such functorial homomorphisms always exist. Thus it is natural to make the following hypothesis $\left(H y p_{A}\right)$ with respect to the given ring $A$.

$\left(\right.$ Hyp $\left._{A}\right)$ For any finite étale extension $A^{\prime} / A$, for any $G$ as above such that $\tilde{Z}$ is smooth, there exist functorial corestriction homomorphisms

$$
\text { Cores }_{A^{\prime} / A}: \mathrm{H}_{a b, e t}^{i}\left(A^{\prime}, G_{A^{\prime}}\right) \rightarrow \mathrm{H}_{a b, e t}^{i}(A, G), i=0,1,
$$

such that if $A^{\prime}=A$ then $N_{A^{\prime} / A}=i d$, and for any tower of finite separable extensions $K / L / k$, with obvious notations $A^{\prime \prime} / A^{\prime} / A$, we have

$$
N_{A^{\prime \prime} / A}=N_{A^{\prime} / A} \circ N_{A^{\prime \prime} / A^{\prime}} .
$$


Assuming $\left(H y p_{A}\right)$, we may also consider the similar notion of Corestricton Principle for the image of $a b_{A^{\prime} / A, e t}^{i}, i=0,1$.

1.3.7. Notice that in many important cases, $\left(H y p_{A}\right)$ above holds for $i=0$, due to Deligne, that we recall briefly below. Recall that for a complex of commutative algebraic $k$-groups $\left(G_{1} \rightarrow G_{2}\right), \mathrm{H}^{0}\left(k, G_{1} \rightarrow G_{2}\right)$ denotes the abelian group of isomorphic objects of the Picard category $\left[G_{1} \rightarrow G_{2}\right]$ (see 1.3). Then, for a finite separable extension $k^{\prime} / k$, it has been shown that there exists an additive trace functor

$$
\operatorname{Tr}_{k^{\prime} / k}:\left[G_{1, k^{\prime}} \rightarrow G_{2, k^{\prime}}\right] \rightarrow\left[G_{1} \rightarrow G_{2}\right]
$$

Also, in [De], Section 2.4.7, there has been established a quasi-isomorphism of complexes

$$
(\tilde{Z} \rightarrow Z) \simeq(\tilde{T} \rightarrow T) \simeq(\tilde{G} \rightarrow G),
$$

which gives rise to additive trace functor (called a "norm functor") between such categories

$$
N_{k^{\prime} / k}:\left[\tilde{G}_{k^{\prime}} \rightarrow G_{k^{\prime}}\right] \rightarrow[\tilde{G} \rightarrow G]
$$

which induces a norm homomorphism

$$
\operatorname{Cores}_{k^{\prime} / k}: \mathrm{H}^{0}\left(k^{\prime}, \tilde{G}_{k^{\prime}} \rightarrow G_{k^{\prime}}\right) \rightarrow \mathrm{H}^{0}(k, \tilde{G} \rightarrow G),
$$

which is, in our notation, nothing else than the corestriction $\mathrm{H}_{a b, e t}^{0}\left(k^{\prime}, G_{k^{\prime}}\right) \rightarrow \mathrm{H}_{a b, e t}^{0}(k, G)$. The situation can be generalized to a more general setting (here we replace Spec $k$ by Spec $(A))$.

In particular case, when $k$ is a non-archimedean local field, we may derive the map Cores $_{A^{\prime} / A}$ : $\mathrm{H}_{a b, e t}^{0}\left(A^{\prime}, G_{A^{\prime}}\right) \rightarrow \mathrm{H}_{a b, e t}^{0}(A, G)$ differently as follows. In above notation (see 1.3.2), we have the following exact sequence

$$
\begin{aligned}
1 \rightarrow \mathbf{H}_{a b, e t}^{-1}(A, G) & \rightarrow \mathrm{H}_{e t}^{0}(A, \tilde{G}) \rightarrow \mathrm{H}_{e t}^{0}(A, G) \stackrel{a b_{G, e t}^{0}}{\rightarrow} \mathbf{H}_{e t}^{0}(A, \tilde{G} \rightarrow G) \rightarrow \\
& \rightarrow \mathrm{H}_{e t}^{1}(A, \tilde{G}) \rightarrow \mathrm{H}_{e t}^{1}(A, G) \stackrel{a b_{G, e t}^{1}}{\rightarrow} \mathbf{H}_{e t}^{1}(A, \tilde{G} \rightarrow G) .
\end{aligned}
$$

According to Tits result (Theorem 2.1.1, a) below), we have $\operatorname{Ker}\left(\mathrm{H}_{e t}^{1}(A, \tilde{G}) \rightarrow \mathrm{H}^{1}(k, \tilde{G})\right)=0$. Since $\mathrm{H}^{1}(k, \tilde{G})=0$ due to well-known Kneser - Bruhat -Tits Theorem, we have $\mathrm{H}_{e t}^{1}(A, \tilde{G})=$ 0 . Since $(\tilde{T} \rightarrow T) \simeq(\tilde{G} \rightarrow G)$, we have $\mathbf{H}_{e t}^{0}(A, \tilde{T} \rightarrow T) \simeq \mathbf{H}_{e t}^{0}(A, \tilde{G} \rightarrow G)$. Hence from the exact sequence

$$
\begin{aligned}
1 \rightarrow \mathbf{H}_{e t}^{-1}(A, \tilde{T} \rightarrow T) & \rightarrow \mathrm{H}_{e t}^{0}(A, \tilde{T}) \rightarrow \mathrm{H}_{e t}^{0}(A, T) \stackrel{a b_{T, e t}^{0}}{\rightarrow} \mathbf{H}_{e t}^{0}(A, \tilde{T} \rightarrow T) \rightarrow \\
& \rightarrow \mathrm{H}_{e t}^{1}(A, \tilde{T}) \rightarrow \mathrm{H}_{e t}^{1}(A, T) \stackrel{a b_{T, e t}^{1}}{\rightarrow} \mathbf{H}_{e t}^{1}(A, \tilde{T} \rightarrow T) .
\end{aligned}
$$


we obtain

$$
\mathrm{H}_{a b, e t}^{0}(A, G) \simeq \operatorname{Coker}\left(\alpha_{A}: \mathrm{H}_{e t}^{0}(A, \tilde{T}) \rightarrow \mathrm{H}_{e t}^{0}(A, T)\right) .
$$

Since for any finite étale extension $A^{\prime} / A$ we have (by 1.3.4.1) a functorial corestriction homomorphisms

$$
\text { Cores }_{A^{\prime} / A}: \mathrm{H}^{0}\left(A^{\prime}, \tilde{T}_{A^{\prime}}\right) \rightarrow \mathrm{H}^{0}(A, \tilde{T}), \text { Cores }_{A^{\prime} / A}: \mathrm{H}^{0}\left(A^{\prime}, T_{A^{\prime}}\right) \rightarrow \mathrm{H}^{0}(A, T),
$$

we may derive another one $\operatorname{Coker}\left(\alpha_{A^{\prime}}\right) \rightarrow \operatorname{Coker}\left(\alpha_{A}\right)$, i.e.,

$$
\operatorname{Cores}_{A^{\prime} / A}: \mathrm{H}_{a b, e t}^{0}\left(A^{\prime}, G_{A^{\prime}}\right) \rightarrow \mathrm{H}_{a b, e t}^{0}(A, G)
$$

1.3.8. Proposition. 1) Let $k$ be a field, $A$ a domain with quotient field $k, G$ a reductive A-group scheme. Assume that for finite étale extension $A^{\prime} / A$ with corresponding finite quotient fields extension $k^{\prime} / k$, the corestriction principle holds for the image of homomor-

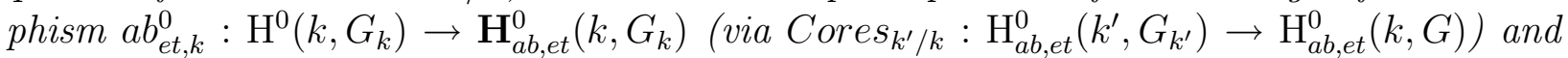
the map $\gamma_{k}: \mathrm{H}_{e t}^{1}(A, \tilde{G}) \rightarrow \mathrm{H}^{1}\left(k, \tilde{G}_{k}\right)$ has trivial kernel. Then the corestriction principle holds for the image of homomorphism ab $b_{e t, A}^{0}: \mathrm{H}^{0}(A, G) \rightarrow \mathbf{H}_{a b, e t}^{0}(A, G)$ (via Cores $_{A^{\prime} / A}$ : $\left.\mathrm{H}_{a b, e t}^{0}\left(A^{\prime}, G_{A^{\prime}}\right) \rightarrow \mathrm{H}_{a b, e t}^{0}(A, G)\right)$.

2) Let $k$ be a local (resp. global) field with the ring of integers $A, \infty$ the set of all archimedean valuations of $k, G$ a reductive A-group scheme, $A^{\prime} / A$ a finite étale extension, and let $k^{\prime}$ be the quotient field of $A^{\prime}$. Assume that in the case of a global field $k$, G has (absolute) strong approximation over $A$, i.e., $G\left(A_{S}\right)$ is dense in $\prod_{v \in S} G\left(k_{v}\right)$ for any finite set $S(\supset \infty)$ of primes of $A$. Then the Corestriction principle holds for the image of $a b_{G, e t}^{0}$.

Proof. 1) We have the following commutative diagram with exact rows for $(A, k)$

$$
\begin{array}{ccccccc}
\tilde{G}(A) & \stackrel{\alpha_{A}}{\rightarrow} & G(A) & \stackrel{a b_{A}}{\rightarrow} & \mathrm{H}_{a b, e t}^{0}(A, G) & \stackrel{\delta_{A}}{\rightarrow} & \mathrm{H}_{e t}^{1}(A, \tilde{G}) \\
\downarrow i d & & \downarrow i d & & \downarrow \gamma_{a b, k} & & \downarrow \gamma_{k} \\
\tilde{G}(k) & \stackrel{\alpha_{k}}{\rightarrow} & G(k) & \stackrel{a b_{k}}{\rightarrow} & \mathrm{H}_{a b, e t}^{0}(k, G) & \stackrel{\delta_{k}}{\rightarrow} & \mathrm{H}^{1}(k, \tilde{G})
\end{array}
$$

and similar one for $\left(A^{\prime}, k^{\prime}\right)$ (where we drop the subscrip to denote the base extension for simplicity)

$$
\begin{aligned}
& \tilde{G}\left(A^{\prime}\right) \stackrel{\alpha_{A^{\prime}}}{\rightarrow} G\left(A^{\prime}\right) \stackrel{a b_{A^{\prime}}}{\rightarrow} \quad \mathrm{H}_{a b, e t}^{0}\left(A^{\prime}, G_{A^{\prime}}\right) \stackrel{\delta_{A^{\prime}}}{\rightarrow} \quad \mathrm{H}_{e t}^{1}\left(A^{\prime}, \tilde{G}_{A^{\prime}}\right) \\
& \downarrow i d \quad \downarrow i d \quad \quad \downarrow \gamma_{a b, k^{\prime}} \quad \downarrow \gamma_{k^{\prime}} \\
& \tilde{G}\left(k^{\prime}\right) \stackrel{\alpha_{k^{\prime}}}{\rightarrow} \quad G\left(k^{\prime}\right) \stackrel{a b_{k^{\prime}}}{\longrightarrow} \quad \mathrm{H}_{a b, e t}^{0}\left(k^{\prime}, G\right) \stackrel{\delta_{k^{\prime}}}{\longrightarrow} \quad \mathrm{H}^{1}\left(k^{\prime}, \tilde{G}\right)
\end{aligned}
$$


Thus we have the following commutative diagram

$$
\begin{aligned}
& \mathrm{H}_{e t}^{1}(A, \tilde{G}) \quad \stackrel{\gamma_{k}}{\longrightarrow} \mathrm{H}^{1}\left(k, \tilde{G}_{k}\right) \\
& \mathrm{H}_{e t}^{1}\left(A^{\prime}, \tilde{G}_{A^{\prime}}\right) \quad \stackrel{\gamma_{k^{\prime}}}{\longrightarrow} \mathrm{H}^{1}\left(k^{\prime}, \tilde{G}_{k^{\prime}}\right) \quad \uparrow \delta_{A} \quad \uparrow \delta_{k} \\
& \uparrow \delta_{A^{\prime}} \quad \uparrow \delta_{k^{\prime}} \quad \mathrm{H}_{a b, e t}^{0}(A, G) \stackrel{\gamma_{a b, k}}{\rightarrow} \mathrm{H}_{a b, e t}^{0}\left(k, G_{k}\right) \\
& \mathrm{H}_{a b, e t}^{0}\left(A^{\prime}, G_{A^{\prime}}\right) \stackrel{\gamma_{a b, k^{\prime}}}{\rightarrow} \mathrm{H}_{a b, e t}^{0}\left(k^{\prime}, G_{k^{\prime}}\right)
\end{aligned}
$$

where $f=$ Cores $_{a b, A^{\prime} / A}$ and $g=\operatorname{Cores}_{a b, k^{\prime} / k}$ exist by Deligne result mentioned above (see 1.3.7.1). Let $x \in G\left(A^{\prime}\right)_{A^{\prime}}$. Let $y:=\delta_{A}\left(f\left(a b_{A^{\prime}}(x)\right)\right)$. To see that $f\left(a b_{A^{\prime}}(x)\right) \in \operatorname{Im}\left(a b_{A}\right)$ is the same to verify that $y=0$, hence it suffices to verify that $\gamma_{k}(y)=0$, since by assumption $\gamma_{k}$ has trivial kernel. But

$$
\begin{aligned}
\gamma_{k}(y) & =\gamma_{k}\left(\delta_{A}\left(f\left(a b_{A^{\prime}}(x)\right)\right)\right. \\
& =\delta_{k}\left(g\left(a b_{k^{\prime}}(x)\right)\right) \\
& =0
\end{aligned}
$$

since the Corestriction principle holds for the image of $a b_{k}$. Thus $y=0$, i.e., $f\left(a b_{A^{\prime}}(x)\right) \in$ $\operatorname{Im}\left(a b_{A}\right)$ as asserted.

2) First assume that $k$ is a local field. Then as in 1.3.7, since $\mathrm{H}_{e t}^{1}(A, \tilde{G})=0$, we conclude as in $1)$. Now we assume that $k$ is a global field. By assumption, $\tilde{G}$ has strong approximation over $A$, thus by [Ha], Corollary 2.3.2, $\mathrm{H}_{Z a r}^{1}(A, \tilde{G})=0$, so from exact sequence $1 \rightarrow \mathrm{H}_{Z a r}^{1}(A, \tilde{G}) \rightarrow$ $\mathrm{H}_{e t}^{1}(A, \tilde{G}) \stackrel{\gamma_{k}}{\rightarrow} \mathrm{H}^{1}(k, \tilde{G})$, (due to Nisnevich, see Theorem 2.1.1 below), we have $\operatorname{Ker}\left(\gamma_{k}\right)=0$. The rest follows again using arguments from 1). Notice that in all cases we have used the fact that over local and global fields, the Corestriction principle holds (see [De], Sec. 2.4, [T1], Thm. 2.5, [T3], Thm. B).

\section{Generalities on class sets (groups) of algebraic groups}

2.1. Serre - Grothendieck conjecture. Let $S$ be an integral, regular, Noetherian scheme with function field $K, G$ a reductive group scheme over $S$, and let $E$ be a $G$-torsor 
over $S$, i.e., a principal homogeneous space of $G$ over $S$ locally trivial for the étale topology of $S$. We say that $E$ is rationally trivial if it has a section over $K$.

First we recall the following conjecture due to Serre and Grothendieck, in the most general form given by Grothendieck. J.-P. Serre and A. Grothendieck in C. Chevalley's Seminar in 1958 ([SCh], Exp. I and Exp. V) and A. Grothendieck in a Bourbaki Seminar [Gr] in 1966 formulated the following conjecture.

Conjecture. ([Gr], Remarque 1.11.) Let $S$ be a locally noetherian regular scheme, $G$ a semisimple group scheme over $S$. Then any $G$-torsor over $S$ which is trivial at maximal points is also locally trivial.

In the case of arbitrary reductive group schemes, the following is a more general formulation of this conjecture (cf. [Ni1], [CTO], p. 97):

(*) If $S$ is as above and $G$ is a reductive $S$-group scheme, then every rationally trivial $G$ torsor is locally trivial for the Zariski topology of $S$.

In other form the conjecture says (cf. [Ni1], [CTO], p. 97)

(**) The following sequence of (pointed) cohomology sets

$$
1 \rightarrow \mathrm{H}_{Z a r}^{1}(S, G) \rightarrow \mathrm{H}_{e t}^{1}(S, G) \rightarrow \mathrm{H}^{1}\left(K, G_{K}\right)
$$

is exact.

Equivalently, it says that

(***) If $S, G$ are as above, $\eta$ is the generic point of $S$ and $A=\mathcal{O}_{x}$ is any local ring at $x \in S \backslash\{\eta\}$, then the natural map of cohomology

$$
\mathrm{H}_{e t}^{1}(A, G) \rightarrow \mathrm{H}^{1}\left(K, G_{K}\right)
$$

has trivial kernel.

Partial results obtained are due to Harder [Ha1], Tits (unpublished, but see [Ni1], Theorem 4.1,) Nisnevich [Ni1], [Ni2], Theorem 4.2, Colliot-Thélène and Sansuc [CTS2] and Colliot-Thélène and Ojanguren [CTO]. We mainly need only the following

2.1.1. Theorem. a) (Tits, cf. [Ni1], Theorems 4.1.) If $A$ is a complete discrete valuation ring with quotient field $K$, and $G$ is a semisimple A-group scheme, then the above conjectures hold.

b) ([Ni1], Théorème 4.2) If $S$ is a regular one-dimensional noetherian scheme and $G$ is a semisimple S-group scheme, then the above conjectures hold.

c) ([Ni1], Théorème 4.5) If $S=$ Spec $R, R$ is a regular local henselian ring and $G$ is $S$ semisimple group scheme, then above conjectures hold. 
2.2. Double classes. We consider the class set of a given flat affine group scheme $G$ of finite type over Dedekind ring $A$ with smooth generic fiber $G_{k}$ over the quotient field $k$ of $A$. Let $X=\operatorname{Spec}(A), \eta \in X$ the generic point of $X, S$ a finite subset of $X_{0}:=X \backslash\{\eta\}$. The ring $\mathbf{A}(S)$ of $S$-adèles is defined as

$$
\mathbf{A}(S):=\prod_{v \in X_{0} \backslash S} A_{v} \times \prod_{v \in S} k_{v}
$$

where $k_{v}$ (resp. $A_{v}$ ) is the completion of $k$ (resp. A) in the $v$-adic topology. We denote by $A_{S}$ the localization of $A$ at $S, \mathbf{A}=$ ind.lim $_{S} \mathbf{A}(S)$ the adèle ring of $k$ (with respect to $A$ !). We recall (see [Ha1], [Ni1], [Ni3], [Ni4]) that the local class set for a prime $v \in X_{0}$ (denoted by $C l_{v}(G)$ ), the $S$-class set, of $G$ with respect to a finite set $S$ of primes of $A$ (denoted by $C l(S, G)$ ), and the class set of $G$ (denoted by $C l_{A}(G)$ ), is the set of double classes

$$
\begin{gathered}
C l_{v}(G):=G\left(A_{v}\right) \backslash G\left(k_{v}\right) / G(k), \\
C l_{A}(S, G)=G(\mathbf{A}(S)) \backslash G(\mathbf{A}) / G(k),
\end{gathered}
$$

and

$$
C l_{A}(G)=G(\mathbf{A}(\emptyset)) \backslash G(\mathbf{A}) / G(k),
$$

respectively. Here $G(k)$ is embedded diagonally into $G(\mathbf{A})$. The double class $G(\mathbf{A}(\emptyset))$.1.G(k) is called the principal class. In the classical case (and notation) of the algebraic groups $G$ defined over a Dedekind ring $A$ with quotient field a global field $k$, which is the ring of integers of $k$, the class set is nothing else than the usual class set of the group $G$, i.e., if $\infty$ is the set of all infinite primes of $A, \mathbf{A}(\infty)$ the set of integral adèles of $\mathbf{A}$ :

$$
\mathbf{A}(\infty):=\prod_{v \notin \infty} A_{v} \times \prod_{v \in \infty} k_{v}
$$

then

$$
C l_{A}(G)=G(\mathbf{A}(\infty)) \backslash G(\mathbf{A}) / G(k),
$$

(cf. [B], [PlR], Chapter VIII, [Ro]).

Especially in the case $G=\mathbf{G}_{m}$, the class set is exactly the ideal class group of the global field $k$. Many other information related with the class number can be found in $[\mathrm{PlR}]$, Chap. VIII and reference therein. In general, class sets contain lot of arithmetic information of the groups under consideration, and it is an important arithmetic invariant for group schemes over $A$. This was one of the main motivations for Nisnevich to introduce a new Grothendieck topology, which was originally called completely decomposed topology and now is called Nisnevich topology. A site with Nisnevich topology is called a Nisnevich site and the corresponding cohomology is called Nisnevich cohomology, denoted by $\mathrm{H}_{N i s}^{i}(X, G)$, where $G$ is a sheaf of groups over a scheme $X$ (see [Ni1] - [Ni4]). The following theorem records most basic properties of Nisnevich cohomology that we need in this paper.

2.2.1. Theorem. Let $X$ be a noetherian scheme of finite Krull dimension $d$. 
1) (Kato - Saito, $[\mathrm{KS}])$ For any sheaf $F$ of abelian groups over $X_{N i s}$, we have $\mathrm{H}_{N i s}^{n}(X, F)=0$, for all $n>d$.

2) ([Ni3], [Ni4]) We have the following exact sequence of cohomology sets for any sheaf of groups $G$ over $X$

$$
\left.1 \rightarrow \mathrm{H}_{N i s}^{1}(X, G) \rightarrow \mathrm{H}_{e t}^{1}(X, G) \rightarrow \mathrm{H}_{N i s}^{0}\left(X, R^{1} f_{*} G\right)\right) \rightarrow 1,
$$

where $f: X_{e t} \rightarrow X_{N i s}$ is canonical projection.

3) ([Ni1], [Ni3], [Ni4]) Let $X$ be a Dedekind scheme Spec(A), G a flat affine group scheme over $X$ of finite type with smooth generic fiber. For a finite set of primes $S, A_{S}$ denotes the localisation of $A$ at $S$. Then we have the following bijections

$$
\begin{gathered}
C l_{v}(G) \simeq \mathrm{H}_{N i s}^{1}\left(A_{v}, G\right), \\
\mathrm{H}_{Z a r}^{1}(A, G) \simeq C l_{A}(G) \simeq \mathrm{H}_{N i s}^{1}(A, G), \\
C l(S, G) \simeq \mathrm{H}_{N i s}^{1}\left(A_{S}, G\right),
\end{gathered}
$$

for all $v$ and finite set of primes $S$.

2.2.2. Remarks. 1) Regarding Theorem 2.2.1, 3), it was shown in [Ha], prior to [Ni1], [Ni3], [Ni4], that there always exists an injection $\mathrm{H}_{Z a r}^{1}(A, G) \hookrightarrow C l_{A}(G)$. Some related results are given in [Gi1] - [GiMB].

2) Some other applications can be found in [T8].

\section{Class groups of algebraic groups}

3.1. Let $k$ be a global field, $A$ a Dedekind ring with quotient field $k, \infty$ the set of infinite primes of $A, \mathbf{A}(\infty)$ the set of integral adèles of $\mathbf{A}$. The problem of computing class sets for a given linear algebraic group $G$ defined over $k$ is a non-trivial one, and depends on the choice of an $A$-integral model $\mathcal{G}_{A}$ of $G$. Namely, take a flat affine $A$-affine group scheme $\mathcal{G}=\mathcal{G}_{A}$ of finite type with generic fiber $G$. Then as in 2.2 , we define the class set for a given $G$ as

$$
C l_{A}(G):=\mathcal{G}(\mathbf{A}(\infty)) \backslash \mathcal{G}(\mathbf{A}) / \mathcal{G}(k)
$$

One of the most interesting cases is when the class set has a natural group structure (i.e., induced from the group structure of $G(\mathbf{A})$ ), which is then called the class group of $G$ (denoted by $\mathcal{G C l}(G)$ as in $[\mathrm{PlR}]$, Chapter VIII). Recall that for a finite set $S$ of primes of $A$, $G$ has weak approximation relative (or with respect) to $S$ if $\mathcal{G}(k)$ is dense in the product of $v$-adic topologies on $\prod_{v \in S} \mathcal{G}\left(k_{v}\right)$. Also (see loc.cit, p. 250), we say that $G$ has strong approximation relative (or with respect) to $S$ (or just $S$-strong approximation) with $S \supset \infty$, if, $\mathcal{G}\left(A_{S}\right)$ is dense in $\prod_{v \in S} \mathcal{G}\left(k_{v}\right)$. Equivalently, the subset $\mathcal{G}(k)$ is dense in $\mathcal{G}\left(\mathbf{A}_{S}\right)$, where $\mathbf{A}_{S}$ denotes the ring of truncated adèles (removing those components belong to $S$ ), or the same, $\mathcal{G}(k) \mathcal{G}_{S}$ is dense in $\mathcal{G}(\mathbf{A})$, where $\mathcal{G}_{S}:=\prod_{v \in S} \mathcal{G}\left(k_{v}\right)$. It is known that the notion of strong approximation 
with respect to $S$ does not depend on the choice of $\mathcal{G}$, and that in this case, $C l_{A}(S, G)=\{1\}$. In the case $S=\infty, G$ is said to have absolute strong approximation over $k$ (or over $A$ ). It is equivalent to saying that $\mathcal{G}\left(A_{W}\right)$ is dense in $\prod_{v \in W} \mathcal{G}\left(k_{v}\right)$ for all finite sets $W \supset \infty$, and in particular we have $C l_{A}(G)=\{1\}$.

It is interesting to see whether the group structure on $\mathcal{G}(\mathbf{A})$ induces a group structure on $C l_{A}(S, G)$. This question has been first addressed by Kneser in [Kn1] - [Kn2], who showed that if $G$ is a connected reductive $k$-group defined over a number field $k$, such that the simply connected covering $\tilde{G}$ of $G$ has the absolute strong approximation, then $C l_{A}(G)$ has a natural structure of finite abelian group. Notice that the arguments in [Kn1] rely on an argument in [Kn2], Hilfsatz 6.2, which are valid for any perfect field $k$, but the proof does not seem to cover the case of non-perfect fields. Then this result has been shown to hold in ([PlR], Prop. 8.8, p. 451), using similar ideas, in the case $k$ is a number field, $G$ is a semisimple algebraic $k$-group.

Our aim in this section is to extend this result (under the assumption on strong approximation with respect to a finite set $S(\supset \infty))$ to the case of connected reductive $k$-groups $G$ over global fields of any characteristic, and we have the following similar property characterizing $C l_{A}(S, G)$ as a finite abelian group. The method of proof is a slight modification of (loc. cit.), by using some arguments due to Deligne [De] and Kneser [Kn1], [Kn2]. The following statements (Theorem 3.2), is important in the proof of our main theorem mentioned in Introduction.

3.2. Theorem. (see [PlR], Prop. 8.8 for semisimple groups, $k$ a number field, [Kn1][Kn2] for connected reductive groups, $k$ a number field)

Let $k$ be a global field, $A$ a Dedekind ring with quotient field $k$. Let $G$ be a connected reductive $k$-group and $\mathcal{G}$ an integral model of $G$ chosen as above. Assume that the simply connected covering $\tilde{G}$ of the derived subgroup $[G, G]$ of $G$ has strong approximation with respect to a finite set of valuations $S \supset \infty$. Then

1) the principal double class $\mathcal{G}(\mathbf{A}(S)) \mathcal{G}(k)$ contains the derived subgroup $[\mathcal{G}(\mathbf{A}), \mathcal{G}(\mathbf{A})]$;

2) the principal double class $\mathcal{G}(\mathbf{A}(S)) \mathcal{G}(k)$ is a normal subgroup of $\mathcal{G}(\mathbf{A})$;

3) the class set $C l_{A}(S, G)$ has a natural structure of a finite abelian group, and we have

$$
C l_{A}(S, G)=\mathcal{G} C l(S, G) \simeq \mathcal{G}(\mathbf{A}) / \mathcal{G}(\mathbf{A}(S)) \mathcal{G}(k) .
$$

Proof. By abuse of notation, and for simplicity, we use also the notation $G(B)$ instead of $\mathcal{G}(B)$, where $B$ is any commutative $A$-algebra.

1) Let $G=G^{\prime} T$, where the product is almost direct, $G^{\prime}$ is semisimple, $T$ is a central $k$ subtorus of $G$ and there is a central $k$-isogeny

$$
1 \rightarrow F \rightarrow \tilde{G} \times T^{\prime} \stackrel{\pi}{\rightarrow} G=G^{\prime} T \rightarrow 1
$$

where $\tilde{G}$ is the simply connected covering of $G^{\prime}$.

It is a standard fact that in a central extension $1 \rightarrow F \rightarrow G \rightarrow H \rightarrow 1$, there exists a homomorphism from the commutator group $[H, H]$ to $G$. From this it follows (cf also observation 
made by Kneser and Deligne (see [Kn1], [De], Sec. 2.0.2)), that in the above exact sequence, $\pi(\tilde{G}(k))$ is a normal subgroup of $G(k)$ with abelian quotient group. In particular,

$$
[G(k), G(k)] \subset \pi(\tilde{G}(k)) .
$$

Moreover, this is true for $G$ considered as a sheaf of groups over some site. Since $\mathbf{A}$ is a $k$-algebra, the above exact sequence can be considered as an exact sequence of $\mathbf{A}$-group schemes, therefore, by considering the flat cohomology we have an exact sequence

$$
1 \rightarrow F(\mathbf{A}) \rightarrow \tilde{G}(\mathbf{A}) \times T^{\prime}(\mathbf{A}) \stackrel{\pi_{A}}{\rightarrow} G(\mathbf{A}) \stackrel{\delta_{A}}{\rightarrow} \mathrm{H}_{\text {flat }}^{1}(\mathbf{A}, F) .
$$

Since the above sequence of groups is exact (see e.g. [Gir], Chap. III, Proposition 3.4.3), and the cohomology group $\mathrm{H}_{\text {flat }}^{1}(\mathbf{A}, F)$ is commutative, it follows that $\operatorname{Im}\left(\pi_{A}\right)$ is a normal subgroup of $G(\mathbf{A})$, containing $[G(\mathbf{A}), G(\mathbf{A})]$. Also, from what has been said, we have

$$
[G(\mathbf{A}), G(\mathbf{A})] \subset \pi_{A}(\tilde{G}(\mathbf{A})) \subset \operatorname{Im}\left(\pi_{A}\right) .
$$

(This has been proved by Kneser in the case of number fields. One may also use the arguments given in [Oe], Chap. II, related with the cohomology of adelic groups, in the case of global function fields.)

By assumption, $\tilde{G}$ has strong approximation with respect to $S$, hence we have $\tilde{G}(\mathbf{A}(S)) \tilde{G}(k)=$ $\tilde{G}(\mathbf{A})$. We show that

$$
\pi_{A}(\tilde{G}(\mathbf{A})) \subset G(\mathbf{A}(S)) G(k)
$$

by showing that

$$
\pi_{A}(\tilde{G}(\mathbf{A}(S))) \subset G^{\prime}(\mathbf{A}(S)) G^{\prime}(k) .
$$

Indeed, let $W$ be a finite set of primes $v$ of $k$ containing $S$, such that $(*)$ defines a short exact sequence of flat $A(W)$-group schemes of finite type (denoted by the same symbols as above) with $\pi$ as central $A(W)$-isogeny. It is clear that we have

$$
\begin{gathered}
\pi_{A}\left(\prod_{v \notin W} \tilde{G}\left(A_{v}\right) \times \prod_{v \in W \cup \infty}\{1\}\right) \subset G^{\prime}(\mathbf{A}(S)) \\
\pi_{A}\left(\prod_{v \in S} \tilde{G}\left(A_{v}\right) \times \prod_{v \notin S}\{1\}\right) \subset G^{\prime}(\mathbf{A}(S)) .
\end{gathered}
$$

Therefore it remains to show that

$$
\pi_{A}\left(\prod_{v \in W \backslash S} \tilde{G}\left(A_{v}\right) \times \prod_{v \notin W \backslash S}\{1\}\right) \subset G^{\prime}(\mathbf{A}(S)) .
$$

Denote by $C l($.$) the operation of taking closure in G(\mathbf{A})$. Let $\mathbf{A}_{S}$ be the ring of $S$-truncated adèles. Since $\tilde{G}$ has $S$-strong approximation over $k, \tilde{G}(k)$ is dense in the adèle topology in the restricted product $G\left(\mathbf{A}_{S}\right)=\prod_{v \notin S}^{\prime}\left(\tilde{G}\left(k_{v}\right), \tilde{G}\left(A_{v}\right)\right.$ ) (the square bracket does not mean taking the commutator group), hence

$$
\prod_{v \in W \backslash S} \tilde{G}\left(A_{v}\right) \times \prod_{v \notin W \backslash S}\{1\} \subset C l(\tilde{G}(k)),
$$


where the closure is taken in $\tilde{G}(\mathbf{A})$. Therefore

$$
\pi_{A}\left(\prod_{v \in W \backslash S} \tilde{G}\left(A_{v}\right) \times \prod_{v \notin W \backslash S}\{1\}\right) \subset \pi_{A}(C l(\tilde{G}(k))) .
$$

Since $\pi_{A}$ is continuous in the adèle topology, which has a countable basis, it follows easily that

$$
\begin{aligned}
\pi_{A}(C l(\tilde{G}(k))) & \subset C l\left(\pi_{A}(\tilde{G}(k))\right) \\
& \subset C l\left(G^{\prime}(k)\right) \\
& \subset C l(G(k)) \\
& \subset G(\mathbf{A}(S)) G(k),
\end{aligned}
$$

since the latter is an open subset of $G(\mathbf{A})$ containing $G(k)$. Therefore we have

$$
\pi_{A}(\tilde{G}(\mathbf{A})) \subset G^{\prime}(\mathbf{A}(S)) G^{\prime}(k)
$$

as required. It follows from above that

$$
\begin{aligned}
{[G(\mathbf{A}), G(\mathbf{A})] \subset \pi_{A}(\tilde{G}(\mathbf{A})) } & =\pi_{A}(\tilde{G}(\mathbf{A}(S)) \tilde{G}(k)) \\
& \subset G(\mathbf{A}(S))) G(k) .
\end{aligned}
$$

2) We show that $G(\mathbf{A}(S)) G(k)$ is a normal subgroup of $G(\mathbf{A})$. Let $g, g_{1} \in G(\mathbf{A}(S)), h, h_{1} \in$ $G(k)$. Then

$$
\begin{array}{rlrl}
(g h)\left(g_{1} h_{1}\right) & =g \cdot g_{1}\left(g_{1}^{-1} \cdot h \cdot g_{1} \cdot h^{-1}\right) h \cdot h_{1} & \\
& =\left(g \cdot g_{1}\right)\left[g_{1}^{-1}, h\right] h \cdot h_{1} & \\
& \in G(\mathbf{A}(S))(G(\mathbf{A}(S)) G(k)) G(k) & & \left.(\text { by } 1) \text { and }\left(^{*}\right)\right) \\
& =G(\mathbf{A}(S)) G(k) ; & \\
(g \cdot h)^{-1} & =g^{-1} \cdot h^{-1}\left(h \cdot g \cdot h^{-1} \cdot g^{-1}\right) & & \left.(\text { by } 1) \text { and }\left(^{*}\right)\right) \\
& =\left(g^{-1} \cdot h^{-1}\right)\left(g_{2} \cdot h_{2}\right) & & \text { (by }(* *)) .
\end{array}
$$

Hence $G(\mathbf{A}(S)) G(k)$ is a subgroup of $G(\mathbf{A})$, and since it contains $[G(\mathbf{A}), G(\mathbf{A})]$, it is a normal subgroup of $G(\mathbf{A})$. 
3) In [Kn1], under the assumption of absolute strong approximation, it has been proved that over a number field $k$, for any $g \in G(\mathbf{A})$, we have

$$
G(\mathbf{A}(\infty)) \cdot g \cdot G(k)=g \cdot G(\mathbf{A}(\infty)) G(k) .
$$

One checks without difficulty that the same argument works in the case of $S$-strong approximation, and also in the case char. $k>0$ (by using 2)). From above we see that $G(\mathbf{A}(S)) G(k)$ is a normal subgroup of $G(\mathbf{A})$, and the double class set

$$
C l_{A}(S, G)=G(\mathbf{A}(S)) \backslash G(\mathbf{A}) / G(k)=G(\mathbf{A}) / G(\mathbf{A}(S)) G(k)=\mathcal{G C l}_{A}(S, G)
$$

is naturally the $S$-class group of $G$, which is finite according to Borel (see [B]) in number field case, Borel - Prasad in global function field case (see [BP], also [Co1], [Co2] in general case of affine $k$-group scheme of finite type).

3.3. Remark. If we replace the condition that $\tilde{G}$ has absolute strong approximation over $k$ by the (obviously weaker) condition

$$
[G(\mathbf{A}), G(\mathbf{A})] \subset G(\mathbf{A}(S)) G(k),
$$

then all the statements of Theorem 3.2 still holds and the proof remains the same.

\section{A norm principle for class groups}

4.1. Assume that the natural group structure exists on the class set of a connected reductive group $G$ defined over a global field $k$, and the same also holds for $G_{k^{\prime}}$ for all finite extension $k^{\prime} / k$. In this case, one may ask if $\mathcal{G C l}(G)$ possesses certain norm map. More precisely, if $k^{\prime} / k$ is a finite separable extension of fields, we ask whether there is a norm homomorphism

$$
N_{k^{\prime} / k}: \mathcal{G C l}\left(G_{k^{\prime}}\right) \rightarrow \mathcal{G C l}(G)
$$

which is functorial in $k^{\prime} / k$, and also in $G$, which coincides with the usual one when $G$ is commutative. In particular, it should be the identity map for $k^{\prime}=k$, and for a towers of separable extensions $k^{\prime \prime} / k^{\prime} / k$, we have

$$
N_{k^{\prime \prime} / k}=N_{k^{\prime} / k} \circ N_{k^{\prime \prime} / k^{\prime}}
$$

With notation as above, in [De], Deligne has introduced the group

$$
\Pi(G):=G(\mathbf{A}) / \pi(\tilde{G}(\mathbf{A})) G(k)
$$


for a connected reductive group $G$ defined over a global field $k$. It is an abelian quotient group of $G(\mathbf{A})$, and it was shown to have a norm homomorphism $N_{k^{\prime} / k}: \Pi\left(G_{k^{\prime}}\right) \rightarrow \Pi(G)$ ([De], Sec. 2.4), which plays a definite role in the study of reciprocity law for canonical models of Shimura varieties. If $\tilde{G}$ has absolute strong appoximation, then the class group $\mathcal{G C l}(S, G)$ is a factor group of $\Pi(G)$ and it is quite possible that in this case, we also have a norm homomorphism $\mathcal{G C l}\left(S^{\prime}, G_{k^{\prime}}\right) \rightarrow \mathcal{G C l}(S, G)$, where $S^{\prime}$ denotes the set of all extensions of $S$ to $k^{\prime}$. In the case of reductive $A$-group schemes we have a property, similar to Theorem 3.2 , for reductive $A$-group schemes, and, under the same assumption, also a norm homomorphism as follows.

Recall that if $k$ is a global function field, under a ring of integers of $k$ we mean the ring of regular functions of an open dense affine subvariety of a smooth projective curve defined over a finite field $\mathbf{F}_{q}$.

4.2. Theorem. (Norm principle for $S$-class groups of algebraic groups.)

Let $k$ be a global field, $A$ the ring of integers of $k, G$ a reductive A-group scheme of finite type. Assume that for a finite set $S$ of primes of $k$, containing the set $\infty$ of archimedean primes, and for the derived subgroup $G^{\prime}=[G, G]$ of $G$, the topological group $\prod_{v \in S} G^{\prime}\left(k_{v}\right)$ is non-compact. For any finite separable extension $k^{\prime} / k, A^{\prime}$ the integral closure of $A$ in $k^{\prime}$, and $S^{\prime}$ the extension of $S$ to $k^{\prime}$, the $S$-class set $C l_{A^{\prime}}(S, G)$ has a natural structure of finite abelian group, and we have a norm homomorphism, functorial in $A$ and $G$

$$
N_{A^{\prime} / A}: \mathcal{G} C l_{A^{\prime}}\left(S^{\prime}, G\right) \rightarrow \mathcal{G C l}_{A}(S, G) .
$$

Proof of Theorem 4.2. We present two proofs of this theorem.

4.2.1. First proof.

4.2.1.1. Claim. Assume that $[G, G]$ is simply connected. Consider the following exact sequence of reductive A-group schemes

$$
1 \rightarrow \tilde{G} \rightarrow G \stackrel{\pi}{\rightarrow} T \rightarrow 1
$$

where $T=G / \tilde{G}$ is an A-torus. Then we have canonical (functorial in A, G) isomorphism of finite abelian groups

$$
\mathcal{G} C l_{A}(S, G) \simeq \mathcal{G C l} l_{A}(S, T)
$$

We know that $\pi$ induces a continuous homomorphism $\pi_{A}: G(\mathbf{A}) \rightarrow T(\mathbf{A})$. We notice that since $\pi$ is defined over $A$, and the class set of $G$ is a class group $\mathcal{G C l}_{A}(S, G), \pi$ induces a homomorphism between class groups

$$
\pi^{\prime}: \mathcal{G C l} l_{A}(S, G) \rightarrow \mathcal{G C l} l_{A}(S, T) .
$$

Let $t=\left(t_{v}\right) \in T(\mathbf{A})$. Let $S_{1}$ be a finite set of finite primes of $A$, such that for $v \notin S_{1}$ we have $t_{v} \in T\left(A_{v}\right)$. We may take $S_{1}$ sufficiently large such that for $S^{\prime}:=\infty \cup S_{1} \cup S$, we have

$$
\mathrm{A}(G) \simeq \mathrm{A}\left(S^{\prime}, G\right):=\prod_{v \in S^{\prime}} G\left(k_{v}\right) / C l(G(k)) \simeq
$$




$$
\simeq \mathrm{A}(T) \simeq \mathrm{A}\left(S^{\prime}, T\right):=\prod_{v \in S^{\prime}} T\left(k_{v}\right) / C l(T(k))
$$

(see the proof of Theorem 2.3 of [T9]). Then $\pi$ induces an isomorphism

$$
\pi_{S^{\prime}}: \mathrm{A}\left(S^{\prime}, G\right) \simeq \mathrm{A}\left(S^{\prime}, T\right),
$$

such that

$$
\left.\pi_{S^{\prime}}^{-1}\left(C l_{S^{\prime}}(T(k))\right)=C l_{S^{\prime}}(G(k))\right)
$$

where the closure is being taken in $\prod_{v \in S \cup \infty} T\left(k_{v}\right)$ (resp. in $\prod_{v \in S \cup \infty} G\left(k_{v}\right)$ ). We can write

$$
t=t_{S^{\prime}} \cdot t_{S^{\prime}}^{\prime}
$$

where

$$
t_{S^{\prime}} \in \prod_{v \in S^{\prime}} T\left(k_{v}\right) \times \prod_{v \notin S^{\prime}}\{1\}, t_{S^{\prime}}^{\prime} \in \prod_{v \notin S^{\prime}} T\left(A_{v}\right) \times \prod_{v \in S^{\prime}}\{1\} .
$$

By Tits result (Theorem 2.1.1 a)), and Kneser - Bruhat - Tits (see [BrT]) about the triviality of the $\mathrm{H}^{1}$ of simply connected groups above, it is clear that $t_{S^{\prime}}^{\prime} \in \operatorname{Im}\left(\pi_{A}\right)$. From the isomorphism above, we can choose $g_{S^{\prime}} \in \prod_{v \in S^{\prime}} G\left(k_{v}\right)$ such that $\pi_{S^{\prime}}\left(g_{S^{\prime}}\right)=t_{S^{\prime}}\left(\bmod . C l_{S^{\prime}}(T(k))\right)$. All these facts show that $\pi$ induces a surjective homomorphism

$$
\pi^{\prime}: \mathcal{G} C l_{A}(S, G) \rightarrow \mathcal{G C l}_{A}(S, T) .
$$

Next we show that $\pi^{\prime}$ is a monomorphism. Let $g=\left(g_{v}\right) \in G(\mathbf{A})$ such that $\pi_{A}(g) \in$ $T(\mathbf{A}(S)) T(k)$, the principal double class of $T(\mathbf{A})$. Let $W$ be a finite set of finite primes of $A$ such that for $v \notin W$ then $g_{v} \in G\left(A_{v}\right)$. Assume that $S^{\prime} \supset S \cup W$ is sufficiently large so that $\mathrm{A}\left(S^{\prime}, G\right)=\mathrm{A}(G)=\mathrm{A}(T)=\mathrm{A}\left(S^{\prime}, T\right)$. Then we write $k_{S^{\prime}}:=\prod_{v \in S^{\prime}} k_{v}, k_{\infty}:=\prod_{v \in \infty} k_{v}$, $\pi_{A}(g)=t_{S^{\prime}} t_{f} t_{k} \in T(\mathbf{A}(S)) T(k)$,

$$
t_{k} \in T(k), t_{S^{\prime}} \in T\left(k_{S^{\prime}}\right) \times \prod_{v \notin S^{\prime}}\{1\}, t_{f} \in \prod_{v \notin S^{\prime}} T\left(A_{v}\right) \times \prod_{v \in S^{\prime}}\{1\} .
$$

As we notice above, $t_{f} \in \operatorname{Im} \pi_{A}$, say $t_{f}=\pi_{A}\left(h_{f}\right)$, where

$$
h_{f} \in \prod_{v \notin S^{\prime}} G\left(A_{v}\right) \times \prod_{v \in S^{\prime}}\{1\} .
$$

By replacing $g=\left(g_{v}\right)$ by $h_{f}^{-1} g$, we may assume that $t_{f}=1$. Thus we have

$$
\pi_{A}(g)=t_{S^{\prime}} t_{k}
$$

Let $t_{S^{\prime}}=t_{1} \cdot t_{2}$, where $t_{1} \in \prod_{v \in S^{\prime} \backslash \infty} T\left(A_{v}\right), t_{2} \in \prod_{v \in \infty} T\left(k_{v}\right)$. The same argument as above shows that

$$
t_{1} \in \operatorname{Im}\left(\pi^{\prime \prime}: \prod_{v \in S^{\prime} \backslash \infty} G\left(A_{v}\right) \rightarrow \prod_{v \in S^{\prime} \backslash \infty} T\left(A_{v}\right)\right) .
$$


Therefore we may assume that $t_{1}=1$, thus also that $\pi_{A}(g)=t_{\infty} t_{k} \in T\left(k_{\infty}\right) T(k)$. Since, as it is well-known, the weak approximation holds for connected $k$-groups with respect to archimedean primes, it follows that $t_{\infty} t_{k} \in C l_{S^{\prime}}(T(k))$. By writing $g=g_{\infty} g_{S \backslash(W \cup \infty)} g_{W \backslash S} g_{W}^{\prime}$, where

$$
\begin{gathered}
g_{\infty} \in G\left(k_{\infty}\right) \times \prod_{v \notin \infty}\{1\}, \\
g_{S \backslash(W \cup \infty)} \in \prod_{v \in S \backslash(W \cup \infty)} G\left(A_{v}\right) \times \prod_{v \notin S \backslash(W \cup \infty)}\{1\}, \\
g_{W \backslash S} \in \prod_{v \in W \backslash S} G\left(k_{v}\right) \times \prod_{v \in S^{\prime}}\{1\}, \\
g_{W}^{\prime} \in \prod_{v \notin S \cup W} G\left(A_{v}\right) \times \prod_{v \in S \cup W}\{1\},
\end{gathered}
$$

to show that $g \in G(\mathbf{A}(S)) G(k)$, we may assume that $g_{W}^{\prime}=1, g_{S \backslash(W \cup \infty)}=1$. Thus we have $\pi_{A}\left(g_{\infty} g_{W \backslash S}\right) \in C l_{S^{\prime}}(T(k))$, hence $g_{\infty} g_{W \backslash S} \in C l_{S^{\prime}}(G(k))$ by our choice $(\#)$. Since $G(\mathbf{A}(S))$ is an open subgroup of $G(\mathbf{A})$, it follows that we have

$$
C l(G(k)) \subset G(\mathbf{A}(\infty)) G(k),
$$

hence

$$
\begin{aligned}
g & =g_{\infty} g_{W \backslash S} \\
& \in C l(G(k)) \prod_{v \notin S} G\left(A_{v}\right) \times \prod_{v \in S}\{1\} \\
& \subset G(\mathbf{A}(S)) G(k) \prod_{v \notin S} G\left(A_{v}\right) \times \prod_{v \in S}\{1\} \\
& \subset G(\mathbf{A}(S)) G(k),
\end{aligned}
$$

where the last inclusion follows from the proof of Theorem 3.2. Thus $g$ has trivial image in the class group as required. (To prove the last inclusion, one may also use the strong approximation assumption and also a result due to Deligne [De], Corollary 2.0.9.)

4.2.1.2. Claim. With above notation and assumptions, we have the following exact sequence of finite abelian groups

$$
1 \rightarrow \mathcal{G C l} l_{A}(Z) \rightarrow \mathcal{G C l} l_{A}(H) \rightarrow \mathcal{G C l} l_{A}(G) \rightarrow 1
$$

Indeed, from the exact sequence

$$
1 \rightarrow Z \rightarrow H \rightarrow G \rightarrow 1
$$

we derive without difficulty the exact sequence on adelic and $k$-points

$$
1 \rightarrow Z(\mathbf{A}) \rightarrow H(\mathbf{A}) \rightarrow G(\mathbf{A}) \rightarrow 1
$$




$$
\begin{gathered}
1 \rightarrow Z(\mathbf{A}(S)) \rightarrow H(\mathbf{A}(S)) \rightarrow G(\mathbf{A}(S)) \rightarrow 1, \\
1 \rightarrow Z(k) \rightarrow H(k) \rightarrow G(k) \rightarrow 1 .
\end{gathered}
$$

and from this the corresponding class groups. (One may also invoke results on Nisnevich cohomology to deduce this (simple) fact. See [Ni4].)

Let

$$
1 \rightarrow \tilde{G} \rightarrow H \rightarrow T \rightarrow 1
$$

be the exact sequence considered before. Due to the functoriality of étale cohomology and also Nisnevich cohomology of tori (or just use the results proved in Sections 2) and the Claim 4.2.1.1, the corestriction (i.e., the norm) homomorphism exist for the class group $\mathcal{G C l}_{A}(Z)$ of $Z$ (denoted by $N_{1}$ ), and for the class group $\mathcal{G} C l_{A}(T)$ of $T$, hence also for $\mathcal{G C l}_{A}(H)$ (denoted by $N_{2}$ ). The following commutative diagram with exact rows (and the map $N_{3}$ )

$$
\begin{aligned}
& 1 \rightarrow \mathcal{G C l}_{A^{\prime}}\left(S^{\prime}, Z_{A^{\prime}}\right) \quad \rightarrow \mathcal{G C l}_{A^{\prime}}\left(S^{\prime}, H_{A^{\prime}}\right) \quad \rightarrow \quad \mathcal{G} C l_{A^{\prime}}\left(S^{\prime}, G_{A^{\prime}}\right) \quad \rightarrow \quad 1 \\
& \downarrow N_{1} \quad \downarrow N_{2} \\
& 1 \quad \rightarrow \quad \mathcal{G C l}_{A}(S, Z) \quad \rightarrow \quad \mathcal{G C l}_{A}(S, H) \quad \rightarrow \quad \mathcal{G C l}_{A}(S, G) \quad \rightarrow \quad 1
\end{aligned}
$$

resulting from this functoriality, shows the existence of the corestriction (norm) map $N_{3}$ for $\mathcal{G C l}_{A}(S, G)$ as required.

4.2.2. Second proof. For simplicity, we assume only that $S=\infty$, and we denote

$$
B=G(\mathbf{A}), C=G(\mathbf{A}(\infty)), D=G(k), E=\tilde{G}(\mathbf{A}), F=\pi(\tilde{G}(\mathbf{A})), J=\tilde{G}(\mathbf{A}(\infty)),
$$

where $\pi: \tilde{G} \rightarrow G^{\prime}=[G, G]$ denotes the canonical projection from simply connected covering $\tilde{G}$ of the semisimple part $G^{\prime}$ of $G$. We prove the following

4.2.2.1. Claim. There exists a norm homomorphism

$$
N: \mathcal{G} C l_{A^{\prime}}\left(G_{A^{\prime}}\right) \rightarrow \mathcal{G C l} l_{A}(G)
$$

which is compatible with the Deligne's norm homomorphism in the sense that the following diagram is commutative

$$
\begin{aligned}
& 1 \quad \rightarrow \operatorname{Ker}\left(q^{\prime}\right) \quad \rightarrow \quad B^{\prime} / F^{\prime} \stackrel{q^{\prime}}{\rightarrow} \mathcal{G} C l_{A^{\prime}}\left(G_{A^{\prime}}\right) \quad \rightarrow 1 \\
& \downarrow q_{1} \quad \downarrow q_{2} \quad \downarrow N_{A^{\prime} / A} \\
& 1 \rightarrow \operatorname{Ker}(f) \quad \rightarrow \quad B / F \quad \stackrel{q}{\rightarrow} \mathcal{G C l}_{A}(G) \quad \rightarrow 1
\end{aligned}
$$


where (.)' means an object is obtained if we pass from $k$ to a finite extension $k^{\prime} / k$, i.e., considered over a finite separable extension $k^{\prime} / k$.

With our assumption on the strong approximation, we know from the proof of Theorem 3.2 , that $C D$ is a normal subgroup of finite index of $B$, and $\mathcal{G C l}_{A}(G)=B / C D$. From [De], Section 2.4 .9 (see also [T1], [T5]), we know that there is a norm homomorphism for the quotient group $B / F$. (In fact, in the case of local fields, and, under our assumption on strong approximation, also that in the case of global fields, one deduces that the Corestriction principle holds for the image of canonical map $a b_{G}^{0}: \mathrm{H}_{e t}^{0}(A, G) \rightarrow \mathrm{H}_{a b, e t}^{0}(A, G)$. (see Proposition 1.3.8.) From this fact, one deduces without difficulty the above mentioned norm homomorphism.) This norm homomorphism is compatible with the Deligne's norm homomorphism for the group $\Pi(G)$, i.e., the following diagram is commutative

$$
\begin{aligned}
& 1 \rightarrow \operatorname{Ker}\left(f^{\prime}\right) \quad \rightarrow \quad B^{\prime} / F^{\prime} \stackrel{f^{\prime}}{\rightarrow} \Pi\left(G_{A^{\prime}}\right) \quad \rightarrow \quad 1 \\
& \downarrow f_{1} \quad \downarrow f_{2} \quad \downarrow f_{3} \\
& 1 \rightarrow \operatorname{Ker}(f) \rightarrow B / F \stackrel{f}{\rightarrow} \Pi(G) \quad \rightarrow \quad 1
\end{aligned}
$$

Indeed, we just need to show that $f_{1}$ is induced from corestriction (norm) homomorphisms previously obtained for algebraic groups over local and global fields as in [T1]. Take a zextension $1 \rightarrow Z \rightarrow H \rightarrow G \rightarrow 1$ (see 1.2.3). By using the surjectivity of the homomorphisms $H(\mathbf{A}) \rightarrow G(\mathbf{A})$ and $H(k) \rightarrow G(k)$, we are reduced to proving the same assertion for $H$, i.e., we may assume $G=H$. But one checks that in this case $\operatorname{Ker}(f)=G(k) \cap \tilde{G}(\mathbf{A})=\tilde{G}(k)$, and the norm homomorphism for Ker $(f)$ is nothing else than the Deligne's norm homomorphism constructed in [De], Section 2.4.

4.2.2.2. We have the following exact sequence of groups

$$
1 \rightarrow \operatorname{Ker}(g) \rightarrow B / F D \rightarrow \mathcal{G C l}_{A}(G) \rightarrow 1 \text {. }
$$

Since there exists a norm homomorphism of $\Pi(G)=B / F D$ compatible with Deligne' norm homomorphism, the proof of the existence of a norm homomorphism of $\mathcal{G C l}_{A}(G)$ compatible with Deligne' norm homomorphism is reduced to that of $\operatorname{Ker}(g)$. Again, as in the previous part, we may assume that $G=H$, i.e., $[G, G]$ is simply connected. In this case one checks that $\operatorname{Ker}(g)=C D / E D$. Since $\tilde{G}$ has absolute strong approximation over $k$, we have

$$
\begin{aligned}
C D / E D & =C D / J D \\
& =C . J D / J D
\end{aligned}
$$




$$
\begin{aligned}
& =C / C \cap J D \\
& =C / J(C \cap D) \\
& =C / J G(A) .
\end{aligned}
$$

Therefore we are reduced to proving the existence of a norm homomorphism for $C / J G(A)$ which is compatible with Deligne' norm homomorphism. We notice that $J$ is a normal subgroup of $C$, and that there exists a norm homomorphism of $C / J$ compatible with Deligne' norm homomorphism (which, for finite primes, follows from Sections 1.3.6 - 1.3.8, and for infinite primes follows from [De] and/or [T1]). By considering the exact sequence

$$
1 \rightarrow \operatorname{Ker}(h) \rightarrow C / J \stackrel{h}{\rightarrow} C / J G(A) \rightarrow 1
$$

we are reduced to proving the same assertion for

$$
\operatorname{Ker}(h)=J G(A) / J=G(A) / J \cap G(A)=G(A) / \tilde{G}(A) .
$$

4.2.2.3. To proceed further with the proof, we consider the exact sequence $1 \rightarrow \tilde{G} \rightarrow G \rightarrow$ $T \rightarrow 1$. We have the following commutative diagram

$$
\begin{array}{ccccc}
\mathrm{H}_{e t}^{0}\left(A^{\prime}, G_{A^{\prime}}\right) \stackrel{\pi^{\prime}}{\rightarrow} & \mathrm{H}_{e t}^{0}\left(A^{\prime}, T_{A^{\prime}}\right) \stackrel{\gamma}{\rightarrow} & \mathrm{H}_{e t}^{1}\left(A^{\prime}, \tilde{G}_{A^{\prime}}\right) \\
& \downarrow N_{A^{\prime} / A} \\
& & & \\
\mathrm{H}_{e t}^{0}(A, G) \stackrel{\pi}{\rightarrow} & \mathrm{H}_{e t}^{0}(A, T) \stackrel{\alpha}{\rightarrow} & \mathrm{H}_{e t}^{1}(A, \tilde{G})
\end{array}
$$

We now show that the Corestriction principle holds for the image of $\pi^{\prime}$, i.e., $N_{A^{\prime} / A}\left(\operatorname{Im}\left(\pi^{\prime}\right)\right) \subset$ $\operatorname{Im}(\pi)$. For this we consider also the following commutative diagram

$$
\begin{aligned}
& \mathrm{H}_{e t}^{1}(A, \tilde{G}) \stackrel{\psi}{\rightarrow} \mathrm{H}^{1}\left(k, \tilde{G}_{k}\right) \\
& \mathrm{H}_{e t}^{1}\left(A^{\prime}, \tilde{G}_{A^{\prime}}\right) \stackrel{\phi}{\rightarrow} \mathrm{H}^{1}\left(k^{\prime}, \tilde{G}_{k^{\prime}}\right) \quad \uparrow \alpha \quad \uparrow \beta \\
& \uparrow \gamma \quad \uparrow \delta \quad \mathrm{H}_{e t}^{0}(A, T) \stackrel{\psi^{\prime}}{\rightarrow} \mathrm{H}^{0}\left(k, T_{k}\right) \\
& \stackrel{f \longrightarrow}{\longrightarrow} \\
& \mathrm{H}_{e t}^{0}\left(A^{\prime}, T_{A^{\prime}}\right) \stackrel{\phi^{\prime}}{\rightarrow} \mathrm{H}^{0}\left(k^{\prime}, T_{k^{\prime}}\right)
\end{aligned}
$$

where the south-east arrows are corestriction homomorphisms. Since $\tilde{G}$ has strong approximation in $S$, the same is true for $\tilde{G}_{A^{\prime}}$ with respect to $S^{\prime}$, the extension of $S$ to $k^{\prime}$. 
The proof of [Ha], Korollar 2.3.2, shows that $\mathrm{H}_{Z a r}^{1}\left(A^{\prime}, \tilde{G}_{A^{\prime}}\right)=0$. Therefore by Nisnevich results (Theorem 2.1.1, b)), the maps $\phi, \psi$ have trivial kernels. Let $x^{\prime} \in \operatorname{Im}\left(\pi^{\prime}\right)$. Then $x^{\prime} \in \operatorname{Ker}(\gamma)=\operatorname{Ker}(\phi \circ \gamma)=\operatorname{Ker}\left(\delta \circ \phi^{\prime}\right)$. By [T1], [T2], the Corestriction principle holds for $\operatorname{Ker}(\delta)$, therefore for $x=\operatorname{Cores}\left(x^{\prime}\right)$ we have $\psi^{\prime}(x) \in \operatorname{Ker}(\beta)$. Hence

$$
\begin{aligned}
\psi\left(\alpha\left(\left(\operatorname{Cores}\left(x^{\prime}\right)\right)\right)\right) & =\beta\left(\psi^{\prime}\left(\operatorname{Cores}\left(x^{\prime}\right)\right)\right) \\
& =\beta\left(\operatorname{Cores}\left(\phi^{\prime}\left(x^{\prime}\right)\right)\right) \\
& =0
\end{aligned}
$$

i.e., $x \in \operatorname{Ker}(\alpha)$, since $\psi$ has trivial kernel.

The proof of Theorem 4.2 (and the one in the Introduction) now follows from above results.

4.3. Some consequences. As a consequence of the proof of Theorem 4.2, we derive the following result, which can be considered as a complement to a description of the class groups given by Nisnevich in the case of semisimple group schemes, or the case of group schemes with semisimple groups as generic fibers) (see [Ni4], Theorem 4.3).

4.3.1. Corollary. With notation and assumption as in Theorem 4.2, there exist welldefined A-tori Z, T, where $Z$ is an induced A-torus, satisfying the following exact sequence of finite abelian groups

$$
1 \rightarrow \mathcal{G C l} l_{A}(S, Z) \rightarrow \mathcal{G C l}_{A}(S, T) \rightarrow \mathcal{G C l} l_{A}(S, G) \rightarrow 1 .
$$

Proof. Take any z-extension

$$
1 \rightarrow Z \rightarrow H \rightarrow G \rightarrow 1
$$

for the reductive $A$-group $G$ (see 1.2.3). Denote by $\tilde{G}$ the derived subgroup of $H$, which is a semisimple simply connected $A$-group scheme, and let $T=H / \tilde{G}$, the $A$-torus quotient. Since $Z$ is an induced $A$-torus, as in Claim 2 of the first proof, we have the corresponding exact sequence for class groups

$$
1 \rightarrow \mathcal{G C l}_{A}(S, Z) \rightarrow \mathcal{G C l}_{A}(S, H) \rightarrow \mathcal{G C l}_{A}(S, G) \rightarrow 1
$$

Also, by Claim 4.2.1.1, we have canonical isomorphism of finite abelian groups

$$
\mathcal{G} C l_{A}(S, H) \simeq \mathcal{G} C l_{A}(S, T)
$$

Thus we obtain the exact sequence desired. 
4.3.2. Corollary. Let $k$ be a global function field or a totally imaginary number field, A a Dedekind ring with quotient field $k, G$ a reductive A-group scheme. Then the class set $C l_{A}(G)$ has a natural structure of a finite abelian group, and we have

$$
C l_{A}(G)=\mathcal{G} C l(G) \simeq G(\mathbf{A}) / G(\mathbf{A}(\infty)) G(k) .
$$

Proof. It follows from the fact, that $G^{\prime}$ has absolute strong approximation over $k$ (see e.g. [PlR], Section 7.4), and from Theorem 3.2 and Theorem 4.2.

4.4. Remarks. 1) It is worth of noticing that the restriction map for the class sets of linear algebraic groups over number fields has been studied before by Rohlfs [Ro], Satz 3.1, in a very general setting. In particular, he studied the map

$$
\text { Res }: G(\mathbf{A}(\infty)) \backslash G(\mathbf{A}) / G(k) \rightarrow G\left(\mathbf{A}_{l}(\infty)\right) \backslash G\left(\mathbf{A}_{l}\right) / G(l),
$$

where $l$ is a finite Galois extension of $k, \mathbf{A}_{l}$ denotes the adèle ring of $l$, and obtained a beautiful expression of the kernel (in the category of pointed sets) of the restriction map Res via Galois cohomology of $G$. Theorem 4.1 can be considered as a complement to this result. It would be nice to extend the results obtained above to the case considered by Rohlfs [Ro], Satz 3.1 and Korollar 3.2.

2) In most of results above, which are proved under the assumption of absolute strong approximation, we may relax this condition by assuming only that the class number of $\tilde{G}$ is equal to 1 . (It would be nice to verify the "Kottwitz principle" ([Ko]) in this case.) Also, one may also reformulate the results for the case of $S$-class groups in an appropriate way, for a finite set $S$ of primes containing $\infty$.

3) It is highly possible that by using similar method of proof, we still have norm homomorphisms for the class group (still under the condition on absolute strong approximation assumption) for any connected reductive $k$-group $G$ (i. e. without assuming that $G$ is a reductive $A$-group scheme).

4) In the case of number fields $k$, [Dem1, Théorème 4.7] (or [Dem2, Théorème 3.9.7]), Demarche gave another proof of Theorem 4.1 by using his results on strong approximation in homogeneous spaces defined over $k$.

Acknowledgements. I would like to thank P. Deligne for an e-mail message related with Section 1.3 , to the referees for pointing out some inaccuracies in the preliminary versions of the paper and P. Gille for some email exchange on the topic of the paper. Thanks are due to the Abdus Salam International Center for Theoretical Physics (Trieste), Max-Planck Institut für Mathematik (Bonn) for the hospitality and support while the work over this paper was carried on. 


\section{References}

[B] A. Borel, Some finiteness properties of adèles groups over number fields. Pub. Math. I. H. E. S. t. 16 (1963), 101 - 126.

[BP] A. Borel and G. Prasad, Finiteness theorems for discrete subgroups of bounded covolume in semisimple groups, Pub. Math. I.H.E.S. t. 69 (1989), 119 - 171; Addedum: ibid, t. 71 (1990), 173 - 177.

[Bo] M. V. Borovoi, The algebraic fundamental group and abelian Galois cohomology of reductive algebraic groups. Preprint Max-Plank Inst., MPI/89-90, Bonn, 1990; also "Abelian Galois Cohomology of Reductive Groups". Memoirs of Amer. Math. Soc. v. 162, 1998.)

[Br] L. Breen, Bitorseurs et cohomologie non-abélienne, in : "Grothendieck Festschrift", v. 1, 401 - 476, Boston - Birkhäuser, 1990.

[BrT] F. Bruhat et J. Tits, Groupes réductifs sur un corps local, Chap. III : Compléments et applications à la cohomologie galoisienne. J. Fac. Sci. Univ. Tokyo, v. 34 (1987), 671 - 688.

[CTO] J. -L. Colliot-Thélène et M. Ojanguren, Espaces principaux homogènes locallement triviaux. Pub. Math. I. H. E. S. t. 75 (1992), 97 - 122.

[CTS] J. -L. Colliot-Thélène and J. - J. Sansuc, Principal homogeneous spaces under flasque tori: Applications. J. Algebra 106 (1987) 148-205.

[Co1] B. Conrad, Finite theorems for algebraic groups over function fields, Compositio Math. v. 148 (2012), 555 - 639. (Cf. also: http://www.math.stanford.edu/ conrad/cosetfinite.pdf.)

[Co2] B. Conrad, Weil and Grothendieck approach to adelic points, Preprint (see: http://www.math.stanford.edu/ conrad/adelictop.pdf).

[De] P. Deligne, Variétés de Shimura : Interprétation modulaire et techniques de construction de modèles canoniques, in : "Automorphic forms, representations and L-functions", Proc. Sym. Pure Math. A. M. S. v. 33 (1979), Part 2, 247 289.

[Dem1] C. Demarche, Le défaut d'approximation forte dans les groupes linéaires connexes. Proc. London Math. Soc. 102 (3), 563-597 (2011).

[Dem2] C. Demarche, "Méthodes cohomologiques pour l'étude des pointes rationnels sur les espaces homogènes". Thèse No. 9596, Université de Paris-Sud. Défense October 23, 2009. 
[Gi1] P. Gille, La R-équivalence sur les groupes réductifs définis sur un corps de nombres. Pub. Math. I. H. E. S., t. 86 (1997), 199 - 235.

[Gi2] P. Gille, "Torseurs sur la droite affine et R-équivalence", Thèse Dr. Sci., Univ. Paris-Sud, 1997. (See also P. Gille, Torseurs sur la droite affine, Trans. Gr. v. 7 (2002), 231 - 245.)

[GiMB] P. Gille and L. Moret-Bailly, Action algébriques des groupes arithmétiques, in Proceedings of the Conférence "Torsors, theory and applications", Edinbourg (2011), Proceedings of the London Mathematical Society, Eds. V. Batyrev and A. Skorobogatov.

[Gir] J. Giraud, "Cohomologie non-abélienne", Grundlehren der Wiss. Math., Springer - Verlag, Bd. 169, 1971.

[GA] C. D. González-Avilés, Abelian class groups of reductive group schemes. Israel J. Math. 196 (2013), no. 1, 175 - 214. (Cf. also: arXiv:1108.3264v1 [math.NT].)

[Gr] A. Grothendieck, Le Groupe de Brauer. II. Théorie cohomologique. Sémin. Bourbaki, Exp. 297, 1965

[Ha] G. Harder, Halbeinfache Gruppenschemata über Dedekindringen. Invent. Math., Bd. 4 (1967), 165 - 191.

[KS] K. Kato and S. Saito, Global class field theory of arithmetic schemes; in : "Applications of algebraic K-theory to algebraic geometry and number theory", Part II (Boulder, Colo., 1983), 255-331, Contemp. Math., 55, Amer. Math. Soc., Providence, RI, 1986.

[Kn1] M. Kneser, Strong approximation, in : " Algebraic groups and Discontinuous subgroups", Proc. Sym. Pure Math. v. 9, A.M.S., 1966, 187 - 196.

[Kn2] M. Kneser, Starken Approximation in algebraischen Gruppen, I, J. für die reine und angew. Math., Bd. 218 (1965) 190 - 203.

[Ko] R. Kottwitz, Stable trace formula: elliptic singular terms. Math. Annalen, Bd. 275 (1986), 365 - 399.

[Ni1] Y. Nisnevich, Espaces homogènes principaux rationellement triviaux et arithmétique des schémas en groupes réductifs sur les anneaux de Dedekind. C. R. Acad. Sci. Paris, Sér. I Math. t.299 (1984), no. 1, 5 - 8.

[Ni2] Y. Nisnevich, Rationally trivial principal homogeneous spaces, purity and arithmetic of reductive group schemes over extensions of two-dimensional regular local rings. C. R. Acad. Sci. Paris, Sér. I Math. t.309 (1989), no. 10, 651 - 655. 
[Ni3] Y. Nisnevich, The completely decomposed topology on schemes and associated spectral sequences in algebraic K-theory, in : "Algebraic K-Theory : Connections with Geometry and Topology", Kluwer Academic Publ. 1989, 241 - 342.

[Ni4] Y. Nisnevich, On certain arithmetic and cohomological invariants of semisimple groups, Preprint, July 1989 (second ed.).

[Oe] J. Oesterlé, Nombre de Tamagawa et groupes unipotents en caractéristique $p$, Invent. Math. v. 78 (1984), 13 - 88.

[Pe] E. Peyre, Galois cohomology in degree three and homogeneous varieties. $K$ Theory, v.15 (1998), 99-145.

$[\mathrm{PlR}] \quad$ V. Platonov and A. Rapinchuk, "Algebraic Groups and Number Theory", Academic Press, 1994.

[Ro] J. Rohlfs, Arithmetisch definierte Gruppen mit Galoisoperation. Invent. Math. 48 (1978), 185-205.

[SCh] Séminaire C. Chevalley, "Anneaux de Chow et applications", Notes polycopieés, I. H. P., Paris, 1958.

[SGA 1] A. Grothendieck et al. : Revêtement étales et groupe fondamental, Séminaire de Géometrie Algébrique du Bois Marie 1960-1961, Lecture Notes in Math. 224, Springer-Verlag, 1971.

[SGA 3] M. Demazure et A. Grothendieck, et al. "Schémas en groupes", Séminaire de Géometrie Algébrique du Bois Marie 1960-1961, Lecture Notes in Math., v. 151 - 153, Springer - Verlag, 1970.

[T1] N. Q. Thăńg, Corestriction Principle in non-abelian Galois cohomology over local and global fields. J. Math. Kyoto Univ. v. 42 (2002), 287 - 304.

[T2] N. Q. Thăńg, Weak Corestriction Principle in non-abelian Galois cohomology. Homology, Homotopy and Applications, v. 5 (2003), 219 - 249.

[T3] N. Q. Thăńg, On Galois cohomology of semisimple groups over local and global fields of positive characteristic, I, Math. Zeit. Bd. 259 (2008), 457 - 470.

[T4] N. Q. Thăńg, Weak approximation, R-equivalence and Whitehead groups. in: "Algebraic K-theory" (Toronto, ON, 1996), Fields Inst. Commun., 16, Amer. Math. Soc., Providence, RI, 1997, 345-354.

[T5] N. Q. Thăńg, Weak approximation, Brauer and $R$-equivalence in algebraic groups over arithmetical fields. II. J. Math. Kyoto Univ. v. 42 (2002), no. 2, $305-316$. 
[T6] N. Q. Thăńg, Equivalent conditions for (Weak) Corestriction principle for nonabelian étale cohomology of reductive group schemes, Vietnam J. Math., v. 38 (2010), 89 - 116 .

[T7] N. Q. Thăńg, Corestriction Principle for non-abelian cohomology of reductive group schemes over arithmetic rings. Proc. Jap. Acad. v. 82 (2006), 147 - 151.

[T8] N. Q. Thăńg, Corestriction Principle for non-abelian cohomology of reductive group schemes over Dedekind rings of integers of local and global fields. Preprint MPI 07-6 (2007).

[T9] N. Q. Thăńg, On Galois cohomology of semisimple algebraic groups over local and global fields of positive characteristic, III. Math. Z. Bd. 275 (2013) , 1287 -1315 . 